\title{
SAR Amplitude Probability Density Function Estimation Based on a Generalized Gaussian Model
}

\author{
Gabriele Moser, Member, IEEE, Josiane Zerubia, Fellow, IEEE, and Sebastiano B. Serpico, Senior Member, IEEE
}

\begin{abstract}
In the context of remotely sensed data analysis, an important problem is the development of accurate models for the statistics of the pixel intensities. Focusing on synthetic aperture radar (SAR) data, this modeling process turns out to be a crucial task, for instance, for classification or for denoising purposes. In this paper, an innovative parametric estimation methodology for SAR amplitude data is proposed that adopts a generalized Gaussian (GG) model for the complex SAR backscattered signal. A closed-form expression for the corresponding amplitude probability density function (PDF) is derived and a specific parameter estimation algorithm is developed in order to deal with the proposed model. Specifically, the recently proposed "method-of-log-cumulants" (MoLC) is applied, which stems from the adoption of the Mellin transform (instead of the usual Fourier transform) in the computation of characteristic functions and from the corresponding generalization of the concepts of moment and cumulant. For the developed GG-based amplitude model, the resulting MoLC estimates turn out to be numerically feasible and are also analytically proved to be consistent. The proposed parametric approach was validated by using several real ERS-1, XSAR, E-SAR, and NASA/JPL airborne SAR images, and the experimental results prove that the method models the amplitude PDF better than several previously proposed parametric models for backscattering phenomena.
\end{abstract}

Index Terms-Generalized Gaussian (GG), parametric estimation, probability density function (PDF), synthetic aperture radar (SAR).

\section{INTRODUCTION}

I N THE context of remotely sensed data analysis, a crucial problem is represented by the need to develop accurate models for the statistics of the pixel intensities. Focusing on synthetic aperture radar (SAR) [1]-[3] data, this modeling process turns out to be a crucial task, for instance, for classification [4]-[7] or denoising [3], [8]-[10] purposes.

From a methodological viewpoint, either parametric or nonparametric estimation strategies can be employed for this task [7]. Specifically, a parametric approach postulates a given mathematical model for each class-conditional probability density function (PDF) and formulates the PDF estimation problem as a

Manuscript received June 23, 2004; revised June 3, 2005. This research was carried out within the framework of the IMAVIS project (Fifth Framework), supported by the European Union (EU). The associate editor coordinating the review of this manuscript and approving it for publication was Dr. Attila Kuba.

G. Moser and S. B. Serpico are with the Department of Biophysical and Electronic Engineering (DIBE), University of Genoa, I-16145, Genoa, Italy (e-mail: gemini@dibe.unige.it; vulcano@dibe.unige.it).

J. Zerubia is with the Projet Ariana, UR INRIA Sophia Antipolis, FR-06902, Sophia Antipolis Cedex, France (e-mail: josiane.zerubia@ sophia.inria.fr).

Digital Object Identifier 10.1109/TIP.2006.871124 parameter estimation one. Several strategies have been proposed in the literature to deal with parameter estimation, such as the maximum likelihood (ML) methodology [3], [7] or the "method of moments" (MoM) [3], [11]. On the contrary, nonparametric PDF estimation approaches do not assume any specific analytical model for the unknown PDF, thus providing more flexibility, although usually presenting internal architecture parameters to be set by the user [7]. In particular, several nonparametric kernel-based estimation and regression architectures have been proposed in the literature that have proved to be effective estimation tools, such as standard Parzen window estimators [7], [12], artificial neural networks (ANNs) [13], [14], or support vector machines (SVMs) [15]-[17].

In this paper, we address the problem of PDF estimation in the specific context of SAR amplitude data analysis. In particular, we focus on parametric estimation strategies and exploit a simple analysis of the distributed SAR scattering in order to develop an innovative parametric model endowed with a consistent estimation strategy.

A standard model for the statistics of the complex signal backscattered by a given ground area, illuminated by a singlelook SAR sensor, adopts a discrete characterization of the phenomena occurring in this area, assuming that the number of scatterers is large, the scatterers are independent and small (as compared with the ground area), the scattering instantaneous phases are independent of the amplitudes and uniformly distributed in $[0,2 \pi]$, and there is no single scatterer dominating the scene [3], [18], [19]. Let us denote by $z$ the complex signal received by the SAR sensor from the ground area $\mathcal{R}$ corresponding to a given pixel, that is

$$
z=x+j y=r \exp (j \theta)=\sqrt{v} \exp (j \theta)
$$

where $x, y, r, v$, and $\theta$ are the real part, the imaginary part, the amplitude, the intensity, and the phase of the complex signal $z$, respectively. The above-mentioned model assumes the presence in $\mathcal{R}$ of a finite set of $n$ independent scattering entities, thus interpreting $z$ as the result of the interference of the corresponding $n$ contributions. In particular, this interference phenomenon motivates the usual noise-like granular aspect of SAR images, known as "speckle" [3], [18]. Specifically, assuming the number $n$ of scatterers to be large, according to the central limit theorem [20], the real and imaginary parts of the backscattered signal are assumed to be jointly Gaussian. In particular, they turn out to be independent, zero-mean Gaussian random variables with equal variances, thus yielding an exponential distri- 
bution for the signal intensity and a Rayleigh distribution for the signal amplitude [3], i.e., ${ }^{1}$

$$
p_{v}(v)=\lambda \exp (-\lambda v), \quad p_{r}(r)=2 \lambda r \exp \left(-\lambda r^{2}\right), \quad r, v \geq 0
$$

where $\lambda$ is a distribution parameter to be estimated according to the image data (e.g., by using ML or MoM [21]). However, real SAR amplitude data often present significantly non-Rayleigh empirical distributions, for instance, exhibiting heavier distribution tails and, thus, requiring a more accurate PDF characterization [3], [19]. Several theoretical models have been proposed in order to improve the estimation quality or to generalize the Rayleigh model to different typologies of SAR data. For example, the Nakagami-Rice distribution has been proposed to model the SAR statistics in the presence of a single strong reflector in a homogeneous clutter [11], [22]. The Gamma distribution has been introduced as a model for a multilook SAR intensity PDF: This model generalizes the exponential distribution of (2) by averaging $L$ single-look exponentially distributed intensities, and is expressed as [3], [23]

$$
p_{v}(v)=\frac{(\lambda L)^{L}}{\Gamma(L)} v^{L-1} \exp (-\lambda L v), \quad v \geq 0
$$

where $\Gamma(\cdot)$ is the standard Gamma function [24]. The corresponding amplitude turns out to be Nakagami-distributed [11], [20], i.e.,

$$
p_{r}(r)=2 r p_{v}\left(r^{2}\right)=\frac{2(\lambda L)^{L}}{\Gamma(L)} r^{2 L-1} \exp \left(-\lambda L r^{2}\right), \quad r \geq 0
$$

The Nakagami-Gamma model has also been extended to the case of multilook polarimetric and interferometric data [25]-[27] and to finite mixtures of Gamma components [28], [29]; its application has been generalized by letting the integer number $L$ of looks be a real positive parameter [interpreted as an "equivalent number of looks" (ENL)] to be estimated together with $\lambda$ according to the image data. Both ML and MoM estimates turn out to be suitable for this estimation task [29], [30].

In [19], a generalized version of the central limit theorem [31] is applied in order to extend the standard scattering model by allowing the real and imaginary parts of the backscattered signal to be jointly symmetric- $\alpha$-stable $(\mathrm{S} \alpha \mathrm{S})$ [32], [33] random variables, thus resulting in the following generalized "heavytailed" Rayleigh model for the amplitude PDF:

$$
p_{r}(r)=r \int_{0}^{+\infty} \rho \exp \left(-\gamma \rho^{\alpha}\right) J_{0}(r \rho) d \rho, \quad r \geq 0
$$

where $\alpha$ and $\gamma$ are positive parameters and $J_{0}(\cdot)$ is the zerothorder Bessel function of the first kind [34], [35]. A momentbased estimation strategy is developed in [19] for this parametric model.

\footnotetext{
${ }^{1}$ Since the random variables $r$ and $v$ are always nonnegative, their PDFs are zero on $(-\infty, 0)$. Therefore, in the following, we shall explicitly define their PDFs only on $[0,+\infty)$.
}

A different approach to SAR scattering modeling is proposed in [36] by assuming the number $n$ of scatterers to be in itself a random variable and the population of scatterers to be controlled by a birth-death-migration process. In this case, a $K$ distribution is obtained for the signal intensity [36]-[39], i.e.,

$$
\begin{aligned}
p_{v}(v)= & \frac{2(\lambda L M)^{(L+M) / 2}}{\Gamma(L) \Gamma(M)} v^{(L+M-2) / 2} \\
& \cdot K_{M-L}\left[2(\lambda L M v)^{1 / 2}\right], v \geq 0
\end{aligned}
$$

where $\lambda, L$, and $M$ are positive distribution parameters, and $K_{\nu}(\cdot)(\nu>0)$ is the $\nu$ th order modified Bessel function of the second kind [34], [35]. The corresponding amplitude distribution is given by

$$
\begin{aligned}
p_{r}(r)= & \frac{4(\lambda L M)^{(L+M) / 2}}{\Gamma(L) \Gamma(M)} r^{L+M-1} \\
& \cdot K_{M-L}\left[2 r(\lambda L M)^{1 / 2}\right], r \geq 0 .
\end{aligned}
$$

The same $K$ distribution is obtained when assuming a multiplicative noise model for the SAR intensity by expressing $v$ as the product of two Gamma-distributed components representing a signal and a noise contribution, respectively [3]. Further extensions of the backscattering modeling approach assuming $n$ as a random variable are proposed in [40]-[43]. MoM turns out to be feasible for the parameter estimation task concerning a $K$-distributed random variable, whereas no closed form is currently available for ML parameter estimation, thus requiring intensive numerical computations or analytical approximations of the PDF itself [3].

Inverse Gaussian (IG) distributions have also been employed to model the amplitude statistics [43]-[45]. A multiplicative model is adopted in [43], assuming a Nakagami distribution for the speckle noise component and a generalized inverse Gaussian (GIG) law for the signal component, thus resulting in the following distribution (named $\mathcal{G}$ distribution) for the amplitude return:

$$
\begin{aligned}
p_{r}(r)= & \frac{2 L^{L}\left(\frac{\lambda}{\gamma}\right)^{\alpha / 2}}{\Gamma(L) K_{\alpha}(2 \sqrt{\lambda \gamma})} r^{2 L-1}\left(\frac{\gamma+L r^{2}}{\lambda}\right)^{(\alpha-L) / 2} \\
& \cdot K_{\alpha-L}\left[2 \sqrt{\lambda\left(\gamma+L r^{2}\right)}\right], r \geq 0
\end{aligned}
$$

where $L$ is the ENL of the Nakagami speckle distribution, and $\alpha, \lambda$, and $\gamma$ are the parameters characterizing the GIG signal distribution. Such a general model includes, as particular cases, the "square root of $K$ " shown in (7) and the $\mathcal{G}^{0}$ distribution, which is endowed in [43] with a moment-based parameter estimation algorithm and successfully applied to the statistics of SAR amplitude data over extremely heterogeneous areas. In [11], the $\mathcal{G}^{0}$ distribution is proved to be equivalent to a Fisher PDF and is applied to the characterization of the statistics of high-resolution SAR imagery over urban areas. A further particular case of the $\mathcal{G}$ model (named the "harmonic branch" $\mathcal{G}^{h}$ ) is proposed in [44] and endowed with a moment-based estimation approach to images of urban areas and mixed terrain. In [45], a normal IG distribution is proposed for the real and imaginary parts of the backscattered complex signal, thus resulting in an amplitude 
PDF formulated as a combination of an IG PDF and a Rice PDF, i.e.,

$$
\begin{aligned}
p_{r}(r)= & \frac{\sqrt{\frac{2}{\pi}} \alpha^{3 / 2} \delta \exp \left(\delta \sqrt{\alpha^{2}-\beta^{2}}\right) r}{\left(\delta^{2}+r^{2}\right)^{3 / 4}} \\
& \cdot K_{3 / 2}\left(\alpha \sqrt{\delta^{2}+r^{2}}\right) I_{0}(\beta r), r \geq 0
\end{aligned}
$$

where $\alpha, \beta$, and $\delta$ are the distribution parameters, and $I_{0}(\cdot)$ is the zeroth order modified Bessel function of the first kind [34], [35]. For this "Rician inverse Gaussian" (RiIG) PDF, a case-specific iterative parameter estimation algorithm is developed in [45].

In addition to the above-mentioned "theoretical" (or, at least, partially theoretical) models, also several empirical models have been used to characterize the statistics of SAR amplitude (or intensity) data, such as the Weibull [46], log-normal [3], [47], or Pearson [48] PDFs.

Several generalizations of the MoM estimation strategy have been proposed in the specific context of SAR amplitude or intensity parametric estimation, focusing attention on parametric families defined on $[0,+\infty)$. Lower, fractional, negative, and complex-order moments have been used in [21] and [49] and applied to the fittings of Gamma and $K$ distributions. The corresponding estimates can be proved to exhibit a reduced variance, as compared with standard MoM estimates [21], although requiring the selection of the optimal order to be employed in the computation of moments. The same approach has been applied to the above-mentioned $\mathrm{S} \alpha \mathrm{S}$ generalized Rayleigh PDF [see (5)] [19] and to the $\mathcal{G}^{0}$ [43] and $\mathcal{G}^{h}$ [44] models. Furthermore, the recently proposed "method-of-log-cumulants" (MoLC) [11], [50] stems from the adoption of the Mellin transform [35] (instead of the usual Fourier transform) in the computation of characteristic functions [20] and from the corresponding generalization of the concepts of moment and cumulant [20]. The method proves to be feasible for most above-mentioned parametric models for SAR amplitude and intensity statistics and to provide smaller variance estimates than MoM for Gamma distributed intensities [11], [29].

In this paper, an innovative parametric model is proposed for SAR amplitude data statistics. In particular, the theoretical circular-Gaussian speckle model is generalized, on a phenomenological basis, by assuming the real and imaginary parts of the backscattered signal to be independent zero-mean generalized Gaussian (GG) [51] random variables and by deriving the resulting amplitude PDF analytically. GGs have been employed for noise modeling in communications, detection, and positioning problems [51], in optical image analysis [52], [53], and in wavelet coefficient statistical modeling [54]-[56]. In this paper, the feasibility of such a model for the characterization of the statistics of SAR amplitude data is investigated.

In Section II, the proposed parametric model is derived, and in Section III, a specific parameter estimation algorithm is developed and proved to be consistent. Section IV reports the results of the application of the proposed parametric approach to the statistical modeling of the grey levels of several real SAR images, proving the method to better fit the amplitude distribution, as compared with the above-mentioned previously proposed theoretical models. Finally, conclusions are drawn in Section V.

\section{GeNERALIZED GAUSSIAN MODEL FOR SAR BACKSCATTERED SIGNALS}

A random variable $u$ is said to be generalized Gaussian if its PDF is given by the following equation [51]:

$$
p_{u}(u)=\frac{\gamma c}{2 \Gamma\left(\frac{1}{c}\right)} \exp \left[-|\gamma(u-m)|^{c}\right], \quad u \in \mathbb{R}
$$

where $c, \gamma>0$ and $m \in \mathbb{R}$. Specifically, $m=E\{u\}$ is the expected value of the generalized Gaussian distribution, $\gamma$ is connected to the variance, thus influencing the dispersion around $m$, and $c$ is a shape parameter dealing with the sharpness of the PDF. Both the Gaussian distribution and the Laplace one can be viewed as particular cases of this general model, and correspond to $c=2$ and $c=1$, respectively.

In this paper, we propose to extend the Gaussian model for the real and imaginary parts of the complex SAR signal $z$ by assuming both components to be distributed according to a generalized Gaussian PDF. Therefore, according to the above-mentioned discrete modeling of the distributed scattering phenomena inside the region $\mathcal{R}$, a natural choice is to accept a symmetrical behavior of $x$ and $y$ [3], thus postulating the same values of the parameters $\{c, \gamma, m\}$ for both random variables. Similarly, we accept the usual assumptions about the independence of these components and about the zero mean, and express their joint PDF as follows:

$$
\begin{aligned}
p_{x y}(x, y) & =p_{x}(x) p_{y}(y) \\
& =\frac{\gamma^{2} c^{2}}{4 \Gamma^{2}\left(\frac{1}{c}\right)} \exp \left[-\gamma^{c}\left(|x|^{c}+|y|^{c}\right)\right], \quad(x, y) \in \mathbb{R}^{2} .
\end{aligned}
$$

This choice allows us to gain a higher flexibility than the standard Gaussian model by explicitly taking into account the possible non-Gaussian sharpness of the joint PDF $p_{x y}$, i.e., by implicitly allowing the amplitude PDF $p_{r}$ to exhibit a nonRayleigh behavior. It is worth noting that the proposed model implicitly accepts the hypothesis, stated by the standard Gaussian model, that no single reflector dominates the scene, i.e., it refers to scattering from distributed targets and not to pure targets.

Specifically, in order to compute a closed-form expression for $p_{r}$, we perform a "rectangular-to-polar" coordinate transformation [20], thus obtaining the following expression for the joint PDF of the signal amplitude $r \geq 0$ and phase $\theta \in[0,2 \pi]$ :

$$
\begin{aligned}
p_{r \theta}(r, \theta) & =r p_{x y}(r \cos \theta, r \sin \theta) \\
& =\frac{\gamma^{2} c^{2} r}{4 \Gamma^{2}\left(\frac{1}{c}\right)} \exp \left[-(\gamma r)^{c}\left(|\cos \theta|^{c}+|\sin \theta|^{c}\right)\right] .
\end{aligned}
$$

Therefore, the corresponding marginal amplitude PDF turns out to be

$$
\begin{aligned}
p_{r}(r) & =\int_{0}^{2 \pi} p_{r \theta}(r, \theta) d \theta \\
& =\frac{\gamma^{2} c^{2} r}{4 \Gamma^{2}\left(\frac{1}{c}\right)} \int_{0}^{2 \pi} \exp \left[-(\gamma r)^{c}\left(|\cos \theta|^{c}+|\sin \theta|^{c}\right)\right] d \theta, r \geq 0
\end{aligned}
$$




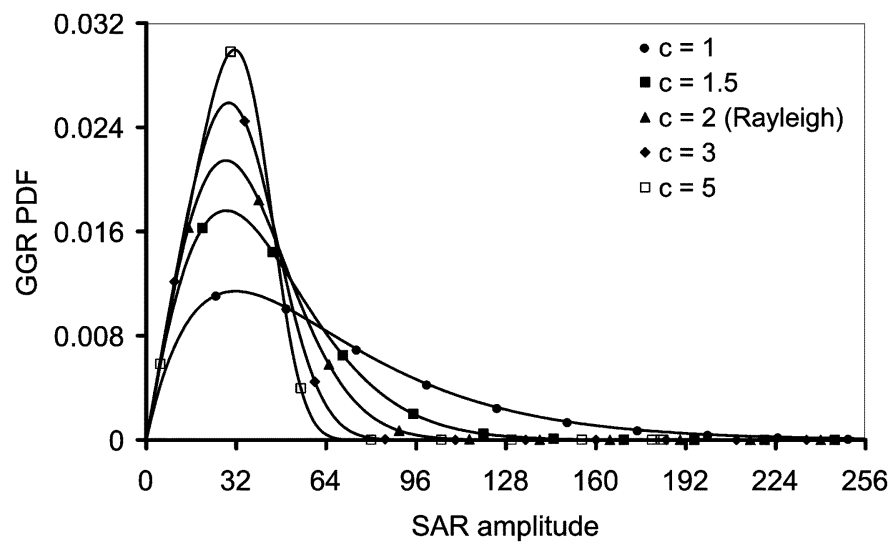

Fig. 1. Plots of several GGR PDFs corresponding to $\gamma=0.025$ and to several distinct values of $c$. The value used for $\gamma$ in these examples has been chosen in order to correctly scale the plotted distributions in the range [0,255] (typical of digital image data).

Since the function $\theta \in \mathbb{R} \longmapsto|\cos \theta|^{c}+|\sin \theta|^{c}$ is $(\pi / 2)$-periodical, we finally obtain

$$
\begin{aligned}
p_{r}(r)= & \frac{\gamma^{2} c^{2} r}{\Gamma^{2}\left(\frac{1}{c}\right)} \\
& \cdot \int_{0}^{\pi / 2} \exp \left[-(\gamma r)^{c}\left(|\cos \theta|^{c}+|\sin \theta|^{c}\right)\right] d \theta, r \geq 0 .
\end{aligned}
$$

We shall refer to this distribution as the generalized Gaussian Rayleigh (GGR) distribution, since it extends the usual Rayleigh-distributed amplitude model by using generalized Gaussian PDFs. Of course, the Rayleigh PDF can be viewed as a particular case of the GGR distribution characterized by $c=2$, i.e.,

$$
\begin{aligned}
p_{r}(r) & =\frac{4 \gamma^{2} r}{\Gamma^{2}\left(\frac{1}{2}\right)} \exp \left[-(\gamma r)^{2}\right] \frac{\pi}{2} \\
& =2 \gamma^{2} r \exp \left[-(\gamma r)^{2}\right], \quad r \geq 0
\end{aligned}
$$

since $\Gamma(1 / 2)=\sqrt{\pi}[24]$.

Fig. 1 compares several GGR PDFs, corresponding to a fixed value of $\gamma$ and to several distinct values of $c$, considering both the Rayleigh PDF (i.e., $c=2$ ) and several non-Rayleigh PDFs. As highlighted in Fig. 1, the GGR parametric family also allows us to take into account the possible "heavy tails" behavior of the amplitude data, corresponding to values of $c$ smaller than 2. A sharper and more impulsive behavior can also be obtained for larger values of $c$ (i.e., $c>2$ ).

\section{ESTIMATION OF THE PARAMETERS OF THE PROPOSED MODEL}

The proposed GGR model for SAR amplitude data is a twoparameter family of PDFs, thus requiring the definition of a suitable estimation procedure, that computes, for a given SAR amplitude image $\mathcal{I}$, the values of $c$ and $\gamma$ that optimally describe the data distribution. From this estimation viewpoint, we consider $\mathcal{I}$ as a set $\mathcal{I}=\left\{r_{1}, r_{2}, \ldots, r_{N}\right\}$ of independent and identically distributed (i.i.d.) samples, drawn from the PDF in (14). This approach is widely accepted in the context of estimation theory [7], [57] and operatively corresponds to discarding, in the estimation process, the contextual information associated with the correlation among neighboring pixels in the image. In addition, the i.i.d. assumption implicitly yields a stationary model for the image data that is accepted as a simplifying hypothesis, although remote-sensing images are typically nonstationary (e.g., due to differences in surface roughness, topography, dielectric constant, or to the presence of distinct landcover typologies in the scene). In particular, this stationarity assumption can be realistic when applied to a set of pixels drawn from the same land-cover class, while it becomes a coarser approximation when applied to a whole image including several land-cover classes.

First, we stress that the GGR model (14) renders the use of a standard ML estimation approach unfeasible. The i.i.d. assumption allows us to obtain the following expression for the log-likelihood function [57] of the image data $\mathcal{I}$

$$
\begin{aligned}
L_{\mathcal{I}}(c, \gamma)= & \sum_{k=1}^{N} \ln p_{r}\left(r_{k}\right) \\
= & 2 N\left[\ln \gamma+\ln c-\ln \Gamma\left(\frac{1}{c}\right)\right]+\sum_{k=1}^{N} \ln r_{k} \\
& +\sum_{k=1}^{N} \ln \int_{0}^{\pi / 2} \exp \left[-\left(\gamma r_{k}\right)^{c}\left(|\cos \theta|^{c}+|\sin \theta|^{c}\right)\right] d \theta .
\end{aligned}
$$

The ML estimation approach would require the numerical maximization of the function $L_{\mathcal{I}}(\cdot, \cdot)$, which would turn out to be a difficult and time-consuming task, due to the presence of the sum of integral contributions in (16). A much more feasible estimation strategy results from adopting the method based on the Mellin transform [11].

\section{A. Parameter Estimation by the Mellin Transform and MoLC}

The MoLC has recently been proposed as a parametric PDF estimation technique for distributions defined on $[0,+\infty)$, and has been explicitly applied in the context of the parametric families usually employed for SAR amplitude and intensity data modeling (e.g., the Nakagami-Gamma and $K$ distributions) [11], [21], [29]. MoLC is based on the generalization of the usual moment-based statistics by applying the Mellin transform for the computations of characteristic functions and moment generating functions (MGFs), instead of the Fourier and Laplace transforms.

Given a generic random variable $u$, the MGF $\Phi_{u}$ of $u$ is defined as the bilateral Laplace transform of the PDF of $u$ [20], i.e.,

$$
\Phi_{u}(s)=\mathcal{L}\left(p_{u}\right)(s)=\int_{-\infty}^{+\infty} p_{u}(u) \exp (s u) d u, \quad s \in \mathbb{C}
$$


where $\mathcal{L}$ is the bilateral Laplace transform operator ${ }^{2}$ on the Lebesgue space $L^{1}(\mathbb{R})$ [58]. The MGF is known to converge and be analytical at least in a vertical strip of the complex plane, and turns out to be implicitly related to the MoM estimation approach. If the interior of the convergence strip contains a neighborhood of the origin, then the $\nu$ th order moment $(\nu=1,2, \ldots)$ can be expressed as

$$
m_{\nu}=E\left\{u^{\nu}\right\}=\Phi_{u}^{(\nu)}(0)
$$

where the superscript denotes a differentiation operator [20]. Related quantities are the characteristic function of $u$ (defined as the Fourier transform of the PDF), the second MGF $\Psi_{u}$ (defined as the complex logarithm of the MGF), and the $\nu$ th-order cumulant $k_{\nu}$ (defined as the $\nu$ th order derivative of the second MGF computed in the origin of the complex plane)

$$
\Psi_{u}(s)=\ln \Phi_{u}(s), \quad k_{\nu}=\Psi_{u}^{(\nu)}(0) .
$$

In particular, the first- and second-order cumulants turn out to be equal to the distribution mean and variance, respectively [20].

The MoM estimates are actually computed by analytically expressing the moments (or the cumulants) of the parametric PDF under investigation as functions of the unknown parameters and by estimating the moments as sample-moments [7], thus formulating the parameter estimation problem as the solution of a (typically, nonlinear) system of equations. In [11] and [50], this approach is specialized to nonnegative random variables (e.g., the SAR amplitude and intensity), corresponding to PDFs defined on $[0,+\infty)$, by redefining MGFs and characteristic functions as Mellin transforms; this results in a more feasible estimation.

Thus, given a nonnegative random variable $u$, the secondkind characteristic function $\phi_{u}$ of $u$ is defined as the Mellin transform [35] of the PDF of $u$, i.e.,

$$
\phi_{u}(s)=\mathcal{M}\left(p_{u}\right)(s)=\int_{0}^{+\infty} p_{u}(u) u^{s-1} d u, \quad s \in \mathbb{C}
$$

where $\mathcal{M}$ is the Mellin transform operator on $L^{1}(0,+\infty)$. Also $\phi_{u}$ is known to converge and be analytical in a vertical strip $\mathcal{S}$ of the complex plane [35]. If the interior of the convergence strip contains a neighborhood of 1 , then the following definitions are formulated by analogy to the Laplace-based case [11]:

- $\quad \nu$ th-order second-kind moment: $\mu_{\nu}=\phi_{u}^{(\nu)}(1), \nu=$ $1,2, \ldots$;

- second-kind second-characteristic function: $\psi_{u}(s)=$ $\ln \phi_{u}(s), s \in \mathcal{S}$;

- $\quad \nu$ th-order second-kind cumulant: $\kappa_{\nu}=\psi_{u}^{(\nu)}(1), \nu=$ $1,2, \ldots$

${ }^{2}$ Actually, the bilateral Laplace operator would involve the exponential $\exp (-s u)$ [35], but, in the context of statistics, the MGF is usually defined with the exponential $\exp (s u)$ as shown in (17). However, this slight modification has no significant impact on the analytical properties of the resulting transform. Hence, hereafter we shall refer to the bilateral Laplace transform defined in (17).
The expressions "log-moments" and "log-cumulants" are also used for the second-kind moments and cumulants, thanks to their relations to the moments of the logarithm of $u$, i.e., [11]

$$
\begin{aligned}
\mu_{\nu} & =E\left\{(\ln u)^{\nu}\right\}, \quad \kappa_{1}=\mu_{1}=E\{\ln u\} \\
\kappa_{2} & =\mu_{2}-\mu_{1}^{2}=\operatorname{Var}\{\ln u\} .
\end{aligned}
$$

Hence, the estimation method of log-cumulants is based on the analytical calculation of log-moments and log-cumulants as functions of the unknown parameters and on the inversion of the resulting equations. Thus, MoLC estimates are obtained from sample-moment estimates of the log-moments or of the log-cumulants by solving a system of nonlinear equations. The numerical solutions of these equations turn out to be feasible and fast for most SAR-specific distributions, such as the Nakagami-Gamma or the $K$ ones, and the MoLC estimates also exhibit lower variances as compared with the usual MoM ones. [29]. However, we stress that, when we deal with nonnegative random variables, we can express the MoM approach itself by using the Mellin transform, since

$$
m_{\nu}=\phi_{u}(\nu+1), \quad \nu=1,2, \ldots
$$

Therefore, if the Mellin transform of the function $p_{u}$ can be computed, both the nonlinear MoM equations and the MoLC ones can be derived.

\section{B. MoLC Parametric Estimation for the GGR Model}

As proved in Appendix A, plugging (14) into the definition (20) of the Mellin transform yields the following expression for the second-kind characteristic function of the GGR model

$$
\phi_{r}(s)=\frac{\Gamma(\lambda s+\lambda)}{\lambda \gamma^{s-1} \Gamma^{2}(\lambda)} \int_{0}^{\pi / 2} \frac{d \theta}{A(\theta, \lambda)^{\lambda s+\lambda}} .
$$

where $\Gamma(\cdot)$ is the usual Gamma function [24], and the auxiliary parameter $\lambda=1 / c$ and the auxiliary function $A:[0, \pi / 2] \times$ $(0,+\infty) \rightarrow \mathbb{R}$, with $A(\theta, \lambda)=|\cos \theta|^{1 / \lambda}+|\sin \theta|^{1 / \lambda}$, have been introduced.

According to (23), $\phi_{r}$ is defined in a neighborhood of 1 , thus allowing the definition of log-cumulants and the application of MoLC. As described in greater details in Appendix A, the computations of the second-kind second characteristic function and of its first and second derivatives yield

$$
\begin{aligned}
& \kappa_{1}=\lambda \Psi(2 \lambda)-\ln \gamma-\lambda \frac{G_{1}(\lambda)}{G_{0}(\lambda)} \\
& \kappa_{2}=\lambda^{2} \Psi(1,2 \lambda)+\lambda^{2} \frac{G_{2}(\lambda) G_{0}(\lambda)-G_{1}(\lambda)^{2}}{G_{0}(\lambda)^{2}}
\end{aligned}
$$

where $\Psi(\cdot)$ is the digamma function [11] (i.e., the logarithmic derivative of the Gamma function), $\Psi(\nu, \cdot)$ is the $\nu$ th order polygamma function [11] (i.e., the $\nu$ th order derivative of the digamma function; $\nu=1,2, \ldots)$, and $G_{\nu}(\cdot)$ is the following integral function:

$$
G_{\nu}(\lambda)=\int_{0}^{\pi / 2} \frac{\ln ^{\nu} A(\theta, \lambda)}{A(\theta, \lambda)^{2 \lambda}} d \theta, \quad \nu=0,1,2, \ldots
$$




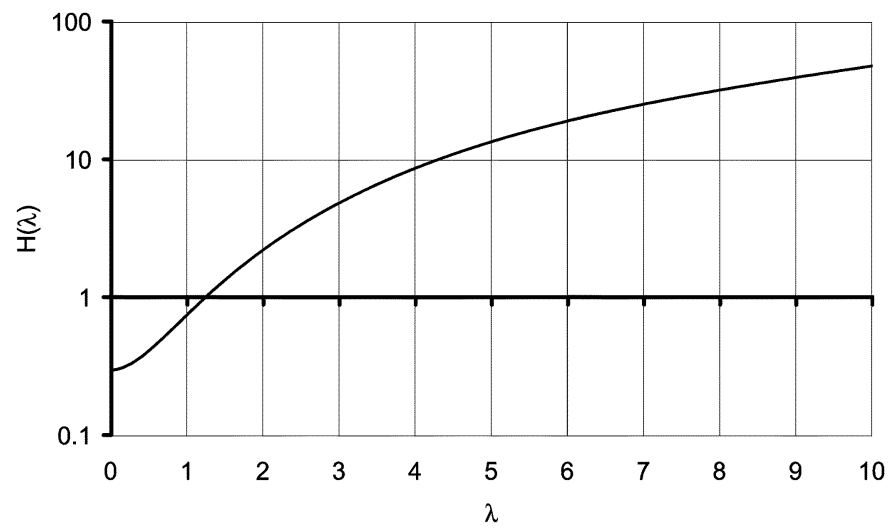

Fig. 2. Logarithmic plot of the $H(\cdot)$ function, representing the right-hand side of (25).

MoLC first computes the following sample-mean and sample-variance estimates of $\kappa_{1}$ and $\kappa_{2}$, according to the image data $\mathcal{I}=\left\{r_{1}, r_{2}, \ldots, r_{N}\right\}[20]$

$$
\hat{\kappa}_{1}=\frac{1}{N} \sum_{k=1}^{N} \ln r_{k}, \quad \hat{\kappa}_{2}=\frac{1}{N-1} \sum_{k=1}^{N}\left(\ln r_{k}-\hat{\kappa}_{1}\right)^{2}
$$

and derives the estimates $\hat{\lambda}$ and $\hat{\gamma}$ of the parameters $\lambda$ and $\gamma$ by solving (24) and (25). We stress that (25) does not contain $\gamma$, thus allowing us to split the nonlinear solution problem into two distinct stages. First, $\lambda$ can be estimated by solving (25); then, the resulting estimate $\hat{\lambda}$ can be plugged into (24) to solve for $\gamma$. The second solution stage can be easily carried out analytically. On the contrary, the first stage requires a numerical solution procedure. However, the function on the right-hand-side of (25), i.e.,

$$
H(\lambda)=\lambda^{2} \Psi(1,2 \lambda)+\lambda^{2} \frac{G_{2}(\lambda) G_{0}(\lambda)-G_{1}(\lambda)^{2}}{G_{0}(\lambda)^{2}}, \quad \lambda>0
$$

is continuous and stricly monotonically increasing (see Fig. 2), thus allowing a simple numerical solution, for instance, by the bisection method [59]. On the other hand, we note that applying MoM would involve computing the first two moments $m_{1}$ and $m_{2}$ [i.e., letting $s=2$ and $s=3$ in (23), respectively], thus leading to more complicated numerical computations.

As shown in Fig. 2, the $H(\cdot)$ function is lower-bounded, i.e., $H(\lambda) \geq H^{*} \simeq 0.296$ for all $\lambda>0$. Hence, if $\hat{\kappa}_{2}<H^{*}$, the equation $H(\lambda)=\hat{\kappa}_{2}$ has no solution. In this case, the GGR model turns out not to be compatible with the empirical data distribution.

Finally, it is worth noting that the developed estimation method for the proposed GGR model also presents significant asymptotic properties. As proved analytically in Appendix B, the MoLC algorithm provides consistent estimates [57] of the true parameters of a GGR distribution. Specifically, assuming the availability of a random sequence $\left\{r_{n}\right\}_{n=1}^{\infty}$ of i.i.d. data samples drawn according to a GGR distribution with parameters $\lambda^{*}$ and $\gamma^{*}$ and denoting by $\hat{\lambda}_{n}$ and $\hat{\gamma}_{n}$ the MoLC estimates of these parameters computed according to the first $n$ data samples $r_{1}, r_{2}, \ldots, r_{n}$, in Appendix $\mathrm{B}$, we prove that the random vector sequence $\left\{\left(\hat{\lambda}_{n}, \hat{\gamma}_{n}\right)\right\}_{n=1}^{\infty}$ converges in probability [20] to the true parameter vector $\left(\lambda^{*}, \gamma^{*}\right)$, i.e.,

$$
\lim _{n \rightarrow+\infty} P\left\{\left|\hat{\lambda}_{n}-\lambda^{*}\right|<\varepsilon,\left|\hat{\gamma}_{n}-\gamma^{*}\right|<\varepsilon\right\}=1 \quad \forall \varepsilon>0 .
$$

Therefore, $\hat{\lambda}_{n}$ and $\hat{\gamma}_{n}$ are consistent estimators of $\lambda^{*}$ and $\gamma^{*}$, respectively.

\section{EXPERIMENTAL RESULTS}

\section{A. Data Sets for Experiments}

The proposed parametric GGR model, endowed with the MoLC estimation strategy described in Section III, was tested on ten real SAR images, and compared with several previously developed theoretical models for SAR amplitude statistics. The first six images used for the experiments were single bands acquired in August 1989 over the agricultural region of Feltwell (U.K.) by a fully polarimetric PLC-band NASA/JPL airborne sensor (for further details on this data set, we refer the reader to [60]). More precisely, all three polarizations $(\mathrm{HH}, \mathrm{HV}$, and $\mathrm{VV}$ ) acquired at band $\mathrm{C}$, the $\mathrm{HV}$ and $\mathrm{VV}$ polarizations acquired at band $\mathrm{L}$, and the $\mathrm{HH}$ polarization acquired at band $\mathrm{P}$ were used. The remaining channels (i.e., L-HH, P-HV, and P-VV) were discarded, because (as reported in [61]) their histograms exhibit strong irregularities. Hereafter, the adopted Feltwell bands will be synthetically denoted by "Feltwell-CHH," "Feltwell-CHV," ..., "Feltwell-PHH."

The other four employed images were:

- a single-look ERS-1 image, acquired in April 1993 over the urban and agricultural regions around Bourges (France);

- $\quad$ an ERS-1 image of the agricultural region of Flevoland (the Netherlands);

- a three-look XSAR scene of a portion of the Swiss territory, including a mountain zone, a portion of a lake, and an urban area (for further details on this image, we refer the reader to [62]);

- a three-look E-SAR image of the area of Oberpfaffenhofen near Munich, Germany.

All the images employed for the experiments were recorded in digital format in the range $[0,255]$, as this scale is usually employed for image processing and classification purposes (e.g., see [19] and [63]). ${ }^{3}$ In Figs. 3 and 4, we show, as examples, the "Feltwell-LHV" and the "Suisse-Lake" images after histogram stretching and/or equalization.

In the experiments reported in Sections IV-B and C, GGR is applied to model the statistics of the above-mentioned images, which, in general, consists of strongly heterogeneous areas presenting several land-cover typologies. If the gray levels corresponding to each land-cover class were modeled as a single statistical population (described, for instance, by a Nakagami or GGR model), the gray-level PDF of the whole image would be a finite mixture density [64]. However, an inspection of the corresponding image histograms (Figs. 5-8) highlights that, due to the strong overlapping of such classes in the (one-dimensional)

${ }^{3}$ However, it is worth noting that a rescaling operation is not critical to GGR modeling: If the amplitude $r$ is GGR-distributed with parameters $c$ and $\gamma$, straightforward calculations allow one to prove that a rescaled amplitude $\alpha r$ $(\alpha>0)$ has again a GGR distribution with parameters $c$ and $\gamma / \alpha$. 


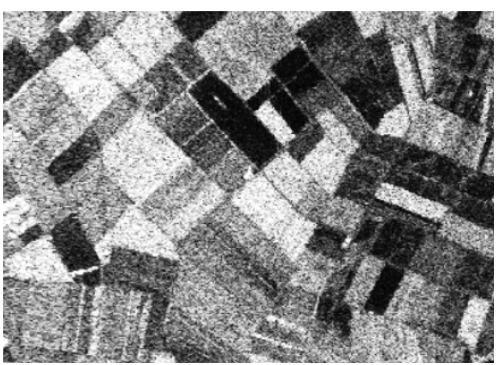

Fig. 3. "Feltwell-LHV" image employed for experiments.

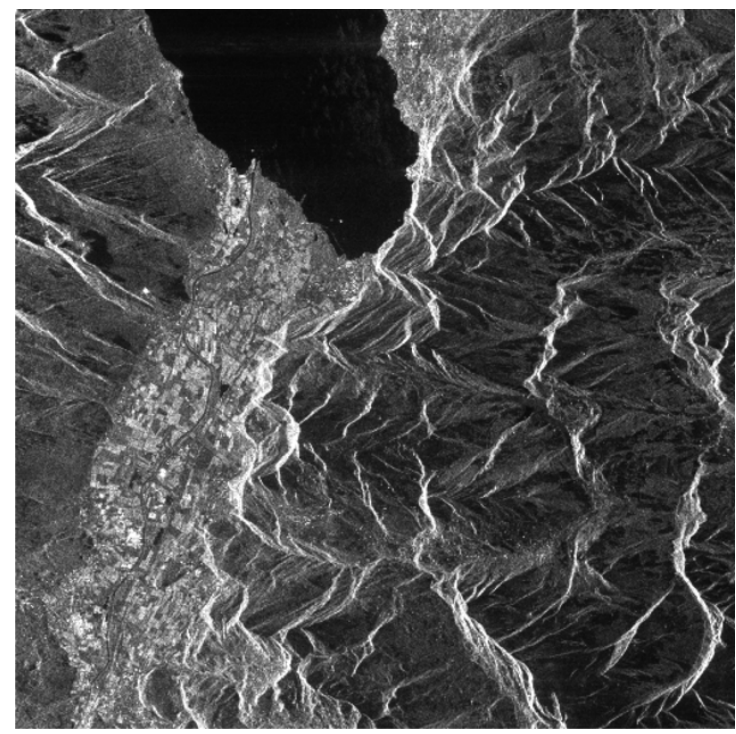

Fig. 4. "Suisse-Lake" image employed for experiments.

feature space, the unconditional gray-level statistics of all the images turn out to be monomodal (although, in general, they could be bimodal or multimodal). Therefore, a single-component PDF (e.g., a single Nakagami or GGR PDF) can be adopted as a model for the image amplitude distribution.

On the other hand, in Section IV-D, we show the result of using the GGR-MoLC estimator to model the statistics of single land-cover classes (specifically focusing on the "Feltwell" data set, for which a ground-truth map is available) in order to analyze the behavior of the proposed method also for homogeneous input areas.

\section{B. PDF Estimation Results}

In order to assess the effectiveness of the proposed parametric PDF estimation algorithm, we have applied the method to the above-mentioned ten images, and have evaluated the estimation results both qualitatively (through a visual comparison between the estimated PDFs and the empirical data distributions, i.e., the image histograms) and quantitatively (i.e., by computing the correlation coefficient between each estimated PDF and the related histogram).

The results have been compared with the ones obtained by several previously developed parametric models for SAR return signals. Dealing with amplitude images, the comparison has involved the Nakagami distribution, the $\mathrm{S} \alpha \mathrm{S}$ generalized Rayleigh distribution (hereafter denoted simply by S $\alpha$ SGR) and the amplitude PDF (7) corresponding to $K$-distributed

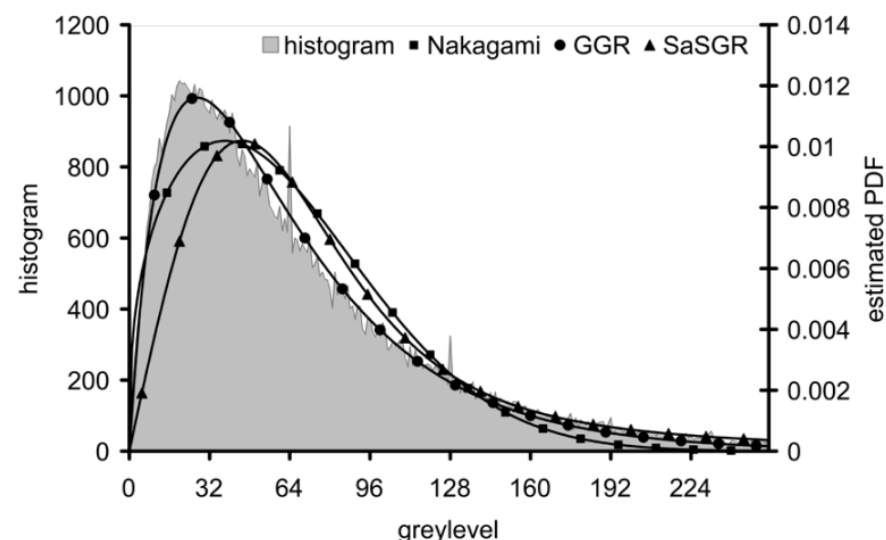

(a)

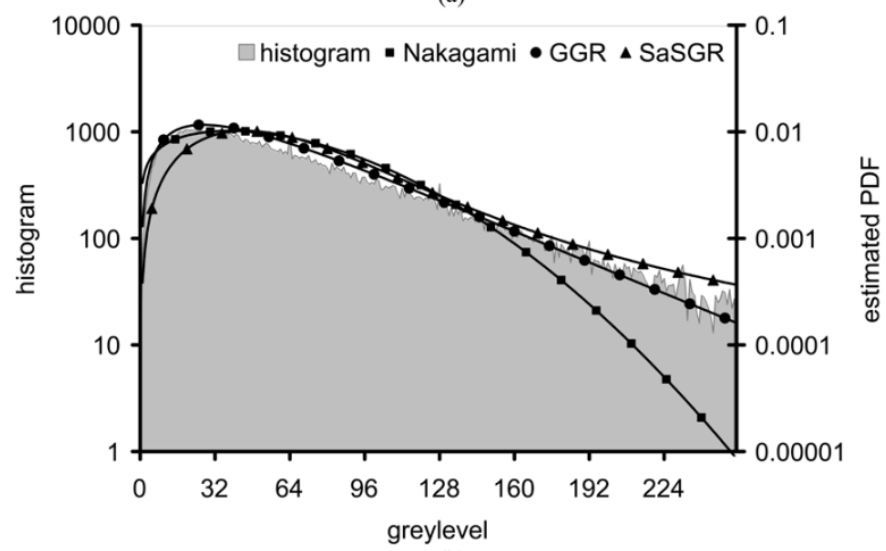

(b)

Fig. 5. Plots of the image histogram and of the estimated GGR, S $\alpha$ SGR, and Nakagami PDFs for the "Feltwell-CHH" data set: (a) linear scale and (b) logarithmic scale.

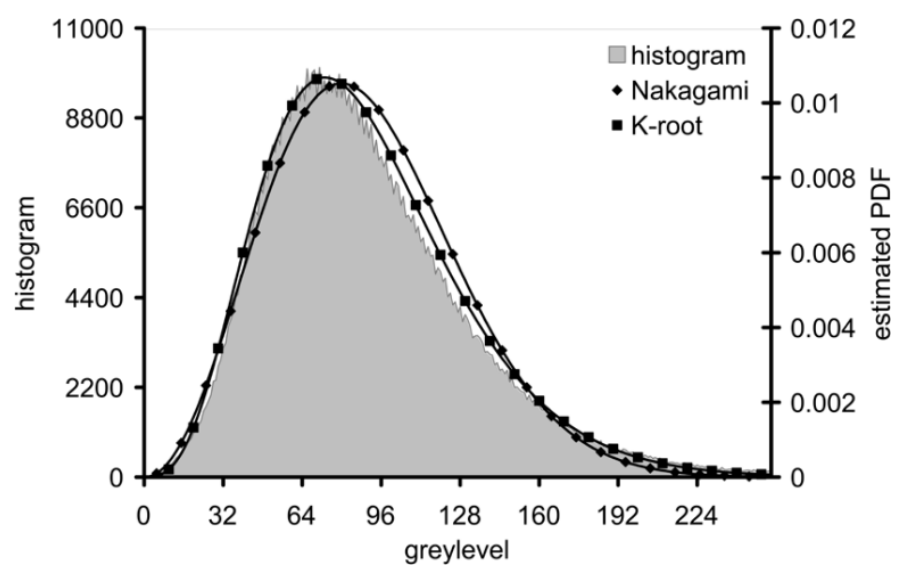

Fig. 6. Plots of the image histogram and of the estimated Nakagami and $K$-root PDFs for the "Flevoland" data set.

intensities (hereafter denoted by " $K$-root"). In the comparison, we have not included the Rayleigh PDF, as it is a particular case of all the above-mentioned models. The MoLC estimation strategy has been adopted for all the considered parametric families, both for homogeneity with the GGR case and for the good estimation properties this algorithm has been proved to exhibit (for instance, for the Nakagami-Gamma model [29]). The resulting correlation coefficients are shown in Table I.

The GGR model turns out to be well defined (i.e., the empirical sample-variance $\hat{\kappa}_{2}$ satisfies the condition $\hat{\kappa}_{2} \geq H^{*}$ ) for 


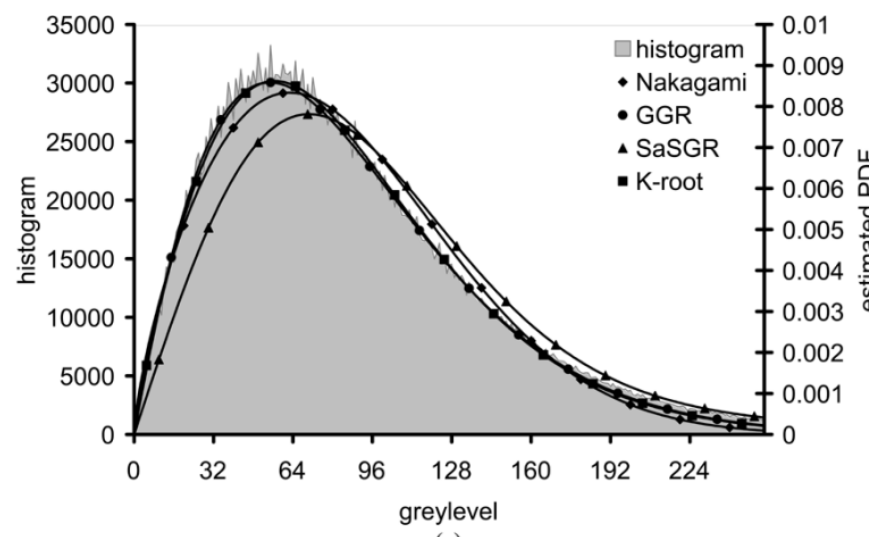

(a)

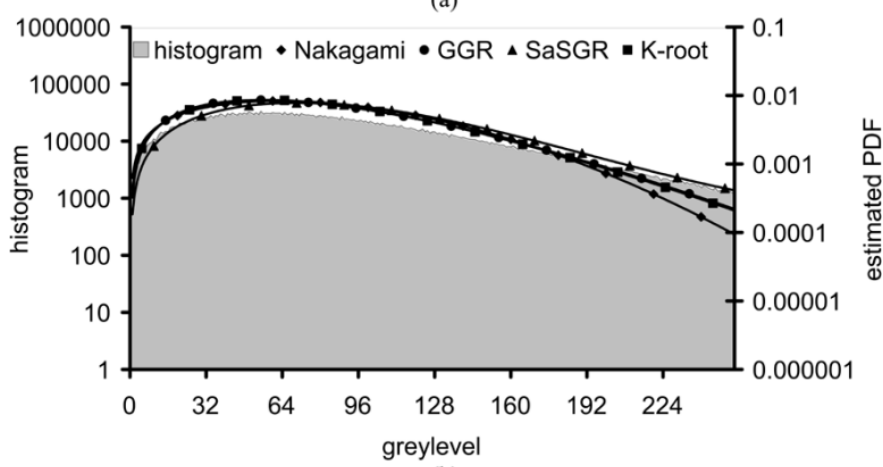

(b)

Fig. 7. Plots of the image histogram and of the estimated GGR, $K$-root, S $\alpha$ SGR, and Nakagami PDFs for the "Bourges" data set: (a) linear scale and (b) logarithmic scale.

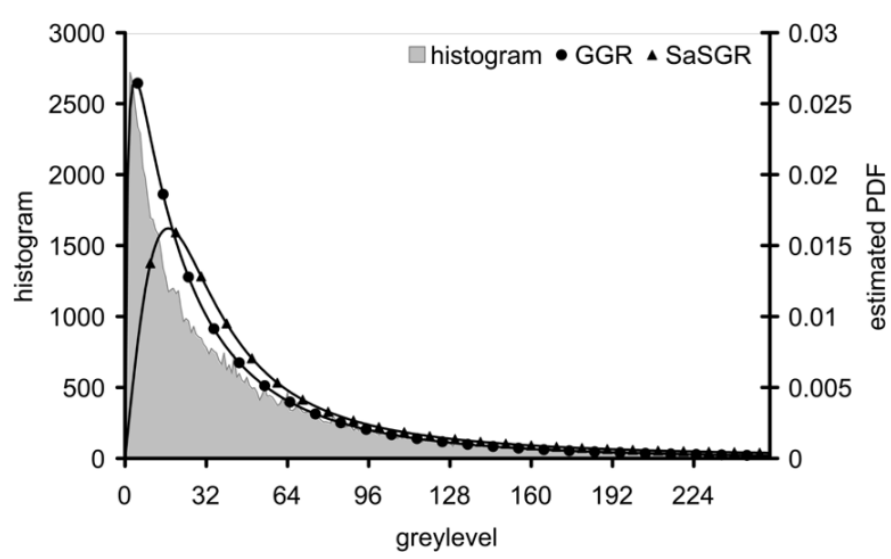

Fig. 8. Plots of the image histogram and of the estimated GGR and S $\alpha$ SGR PDFs for the "Feltwell-LHV" data set.

all the considered images except "Flevoland" and "Oberpfaffenhofen." In all the cases in which the GGR fitting is feasible, except "Bourges," the quantitative correlation coefficients suggest that the proposed parametric estimation approach better fits the data histograms than the other considered parametric models. A visual comparison between the histograms and the plots of the estimated PDFs actually confirms this conclusion. As an example, we show in Fig. 5 the results obtained for "FeltwellCHH."

It should be stressed that it is possible to fit the $K$-root model only to "Bourges," "Oberpfaffenhofen," and "Flevoland." As in the case with GGR, the system of nonlinear equations to be
TABLE I

CORRElATION COEFFICIENTS BETWEEN THE ESTIMATED PDFS AND THE IMAGE HistogRAMS FOR ALL THE CONSIDERED PARAMETRIC FAMILIES AND ALL THE EMPLOYED SAR IMAGES

\begin{tabular}{|c||c|c|c|c|}
\hline \multicolumn{1}{|c||}{ Image } & \multicolumn{4}{c|}{ Parametric model } \\
\cline { 2 - 5 } & GGR & Nakagami & S $\Theta$ SGR & K-root \\
\hline \hline Feltwell-CHH & $99,42 \%$ & $95,80 \%$ & $89,98 \%$ & not defined \\
\hline Feltwell-CHV & $98,95 \%$ & $97,13 \%$ & $88,87 \%$ & not defined \\
\hline Feltwell-CVV & $99,11 \%$ & $94,56 \%$ & $87,54 \%$ & not defined \\
\hline Feltwell-LHV & $98,74 \%$ & $94,14 \%$ & $75,55 \%$ & not defined \\
\hline Feltwell-LVV & $99,28 \%$ & $93,43 \%$ & $84,96 \%$ & not defined \\
\hline Feltwell-PHH & $98,61 \%$ & $92,54 \%$ & $81,57 \%$ & not defined \\
\hline Bourges & $98,97 \%$ & $98,97 \%$ & $95,57 \%$ & $99,77 \%$ \\
\hline Flevoland & not defined & $98,70 \%$ & $88,32 \%$ & $99,77 \%$ \\
\hline Suisse-Lake & $99,63 \%$ & $97,90 \%$ & $94,30 \%$ & not defined \\
\hline Oberpfa enhofen & not defined & $99,88 \%$ & $91,00 \%$ & $99,86 \%$ \\
\hline
\end{tabular}

solved in order to compute the parameter estimates for $K$-root can have no solutions for specific combinations of the values of the sample-log-cumulants [61]. However, the experiments suggest a complementarity between the proposed GGR model and the usual $K$-root one, since, for all the images except "Bourges," only one of the two parametric PDFs turns out to be feasible. In particular, the histogram of "Flevoland" is characterized by poor contents at small gray-level values (Fig. 6), and as reported in [61], "Oberpfaffenhofen" has a very narrow dynamics range, both conditions yielding too small values of the empirical sample-variance of the logarithm of the greylevels to allow the application of GGR. However, in both cases, $K$-root performs very well in fitting the histograms of these images, both from the viewpoint of the correlation coefficients (see Table I) and from the viewpoint of the visual comparison between the histograms and the estimated PDFs (see, for instance, Fig. 6). Furthermore, for the "Bourges" image, which allows the application of both models, the results obtained by GGR and $K$-root are good and quite similar (see Fig. 7). On the contrary, as pointed out by Table I and Fig. 7, both Nakagami and S $\alpha$ SGR yield worse estimation performances, thus proving to be less effective amplitude PDF models than GGR and $K$-root.

We note that, when the image histogram exhibits a very "peaky" distribution, i.e., as in the "Feltwell-LHV" and "Feltwell-PHH" cases (see, for instance, Fig. 8), although providing the best estimation performances among the considered models (see Table I), GGR yields a visually biased result, as it does not accurately model the narrow peak of the distribution. In order to improve the estimation accuracy for such "peaky" distributions, an even higher flexibility of the estimation model would be required, which might be obtained, for instance, by further generalizing GGR by using different values of the parameters $c$ and $\gamma$ of the two generalized Gaussian components of the complex signal.

An analysis of the parameter estimates computed by MoLC for the eight images for which GGR is well defined (see Table II) points out the greater usefulness of the proposed generalized Gaussian model as compared with the standard Gaussian one. For all the images, the estimate $\hat{c}=1 / \hat{\lambda}$ of the shape parameter $c$ turned out to be far smaller than 2 (often even smaller than 1), thus highlighting the strongly non-Gaussian behavior of the signal $z$ and stressing the need to take into account the presence of heavy distribution tails. It is also interesting to note that the 
TABLE II

PARAMETER Estimates Provided By MOLC (WITH $\hat{c}=1 / \hat{\lambda}$ ) FOR THE PROPOSED GGR MODEL APPLIED TO ALL THE IMAGES USED. "FlEVOLAND" AND "OBERPFAFFENHOFEN" ARE NOT CONSIDERED Here, Since the Estimation Process Yields no Solutions FOR THESE IMAGES (SEE TABLE II)

\begin{tabular}{|c||c|c|}
\hline \multicolumn{1}{|c||}{ Image } & $\hat{c}$ & $\hat{\gamma}$ \\
\hline \hline Feltwell-CHH & 0,8576 & 0,0322 \\
\hline Feltwell-CHV & 0,6903 & 0,0506 \\
\hline Feltwell-CVV & 0,7633 & 0,0444 \\
\hline Feltwell-LHV & 0,3683 & 1,0947 \\
\hline Feltwell-LVV & 0,6032 & 0,0931 \\
\hline Feltwell-PHH & 0,4635 & 0,4817 \\
\hline Bourges & 1,3722 & 0,0132 \\
\hline Suisse-Lake & 1,0376 & 0,0271 \\
\hline
\end{tabular}

TABLE III

CORRELATION COEFFICIENTS BETWEEN THE ESTIMATED PDFS AND THE IMAGE Histograms FOR THE $\mathcal{G}^{h}$ AND RIIG PARAMETRIC FAMILIES AND ALL THE EMPLOYED SAR IMAGES

\begin{tabular}{|c||c|c|}
\hline \multicolumn{1}{|c||}{ Image } & \multicolumn{2}{c|}{ Parametric model } \\
\cline { 2 - 3 } & $\mathcal{G}^{h}$ & RiIG \\
\hline \hline Feltwell-CHH & $98.26 \%$ & $97.46 \%$ \\
\hline Feltwell-CHV & $98.56 \%$ & $93.80 \%$ \\
\hline Feltwell-CVV & $97.54 \%$ & $97.82 \%$ \\
\hline Feltwell-LHV & $97.06 \%$ & $94.00 \%$ \\
\hline Feltwell-LVV & $97.06 \%$ & $97.98 \%$ \\
\hline Feltwell-PHH & $96.74 \%$ & $98.41 \%$ \\
\hline Bourges & $99.47 \%$ & $95.08 \%$ \\
\hline Flevoland & $99.35 \%$ & $99.21 \%$ \\
\hline Suisse-Lake & $98.21 \%$ & $95.49 \%$ \\
\hline Oberpfaffenhofen & $99.64 \%$ & $99.86 \%$ \\
\hline
\end{tabular}

estimate $\hat{\gamma}$ of the parameter $\gamma$ exhibits, for "Feltwell-LHV" and "Feltwell-PHH," much larger values than for the other images. This may be interpreted as a consequence of the fact that both "Feltwell-LHV" and "Feltwell-PHH" exhibit the above-mentioned "peaky" behavior in the data distribution, thus presenting a proportionally lower variance than the other images. Plugging expression (23) of the second-kind characteristic function of the GGR PDF in relation (22) between the moments and the Mellin transform, we obtain $\operatorname{Var}\{r\}=m_{2}-m_{1}^{2}=\phi_{r}(3)-$ $\phi_{r}(2)^{2} \propto \gamma^{-2}$. Therefore, for images characterized by "peaky" histograms, $\operatorname{Var}\{r\}$ has a small value, and so a correspondingly large value is expected for the parameter $\gamma$.

\section{Comparison With IG Models}

A further experimental comparison was made between GGR and two recently proposed parametric amplitude models based on IG distributions [43]. Specifically, the $\mathcal{G}^{h}$ distribution proposed in [44] [which is a special case of the PDF in (8)] and the RiIG PDF used in [45] and expressed by (9) were included in the comparison (see Section I). An application of MoLC to these two models has not been formulated yet. On the contrary, the use of fractional moments is proposed in [44] to estimate the parameters of $\mathcal{G}^{h}$, and a case-specific iterative parameter estimation algorithm is developed in [45] for RiIG. Therefore, these two parameter estimation approaches were adopted in the present experiment, and the corresponding PDF estimates were compared with the ones obtained by the proposed GGR-MoLC strategy. The results of the applications of $\mathcal{G}^{h}$ and RiIG to the above-mentioned ten images are presented in Table III.
TABLE IV

CORRELATION COEFFICIENTS BETWEEN THE EsTIMATED PDFS AND THE DATA HISTOGRAMS FOR ALL THE CONSIDERED PARAMETRIC FAMILIES AND ALL THE FIVE ClASSES IN THE "FelTwELL-CHV” IMAGE

\begin{tabular}{|c||c|c|c|c|}
\hline \multirow{2}{*}{ Class } & \multicolumn{4}{|c|}{ Parametric model } \\
\cline { 2 - 5 } & GGR & Nakagami & S $\alpha$ SGR & K-root \\
\hline \hline carrots & $89.99 \%$ & $87.72 \%$ & $83.72 \%$ & $89.95 \%$ \\
\hline potatoes & $89.01 \%$ & $87.30 \%$ & $82.66 \%$ & $88.88 \%$ \\
\hline bare soil & $95.60 \%$ & $89.88 \%$ & $84.85 \%$ & not defined \\
\hline stubble & $97.48 \%$ & $94.73 \%$ & $89.24 \%$ & not defined \\
\hline sugar & $94.38 \%$ & $93.39 \%$ & $89.14 \%$ & $95.55 \%$ \\
\hline
\end{tabular}

TABLE V

CORRELATION COEFFICIENTS BETWEEN THE ESTIMATED PDFs AND THE DATA HistOGRAMS FOR THE "SUGAR" ClASS, FOR ALL THE CONSIDERED PARAMETRIC FAMILIES AND FOR ALL THE "FELTWELL" IMAGES

\begin{tabular}{|c||c|c|c|c|}
\hline \multicolumn{1}{|c||}{ Image } & \multicolumn{4}{c|}{ Parametric model } \\
\cline { 2 - 5 } & GGR & Nakagami & S $\alpha$ SGR & K-root \\
\hline \hline Feltwell-CHH & $91.89 \%$ & $89.76 \%$ & $85.69 \%$ & $91.67 \%$ \\
\hline Feltwell-CHV & $94.38 \%$ & $93.39 \%$ & $89.14 \%$ & $95.55 \%$ \\
\hline Feltwell-CVV & $93.61 \%$ & $92.05 \%$ & $87.77 \%$ & $93.92 \%$ \\
\hline Feltwell-LHV & $96.78 \%$ & $94.89 \%$ & $91.61 \%$ & not defined \\
\hline Feltwell-LVV & $96.35 \%$ & $94.48 \%$ & $90.84 \%$ & $96.79 \%$ \\
\hline Feltwell-PHH & $97.19 \%$ & $95.06 \%$ & $91.58 \%$ & not defined \\
\hline
\end{tabular}

Both IG-based models provided good estimation accuracies for all the ten images considered, though a global better result was obtained by $\mathcal{G}^{h}$ than by RiIG. However, a comparison between Tables I and III shows that, for almost all the eight images for which GGR is well defined, a higher correlation coefficient between the estimated PDF and the image histogram was obtained by GGR than by $\mathcal{G}^{h}$ or RiIG, although the differences between the correlation coefficients of these approaches were small for some images (e.g., GGR and $\mathcal{G}^{h}$ provided similar results on "Feltwell-CHV" and "Bourges"). Analogously, for the three images for which $K$-root was well defined, the correlation coefficients obtained by $K$-root were slightly higher than the ones yielded by $\mathcal{G}^{h}$ and RiIG (an exception was "Oberpfaffenhofen," for which $K$-root and RiIG achieved the same value of the correlation coefficient).

\section{PDF Estimation Over Homogeneous Areas}

In this section, we focus on the use of GGR to model the statistics of homogeneous areas. A ground-truth map was available for the "Feltwell" data set, presenting five main classes: "carrots" (1781 samples), "potatoes" (2295 samples), "bare soil" (896 samples), "stubble" (2441 samples), and "sugar" (3531 samples). For instance, focusing on the "Feltwell-CHV" image, the proposed GGR-MoLC parametric estimation algorithm was applied to the set of samples corresponding to all the classes, and compared with the Nakagami, S $\alpha$ SGR, and $K$-root distributions (again endowed with MoLC estimates; see Table IV). On the other hand, focusing, for instance, on the "sugar" class, the same comparative experiment was repeated over all six "Feltwell" bands (Table V).

In both cases, for each set of samples considered, GGR turned out to be well defined and obtained a good value of the correlation coefficient between the estimated PDF and the histogram of the gray levels related to the set of samples, also obtaining a 


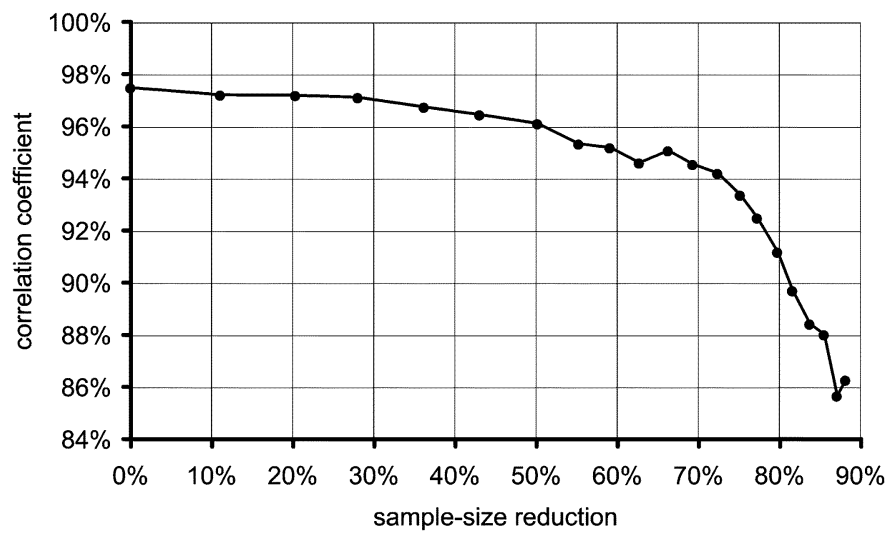

Fig. 9. Plot of the correlation coefficient between the estimated PDF and the data histogram as a function of the (percent) sample-size reduction in the subsampled "stubble" class in the "Feltwell-CHV" image.

higher correlation coefficient than Nakagami and S $\alpha$ SGR (Tables IV and V). On the contrary, $K$-root, applied to the five classes in "Feltwell-CHV," was feasible only for three classes (namely, "carrots," "potatoes," and "sugar"; see Table IV) and was applicable to the "sugar" areas in four of the six "Feltwell" bands (Table V). Similar results were obtained on the other four classes (e.g., in the application to the "stubble" areas, $K$-root was not feasible in any "Feltwell" band, whereas GGR obtained correlation coefficients above $96.6 \%$ in all the six bands). However, as already noted with regard to the whole images, when both GGR and $K$-root were well defined, the results provided by such models were similar: In particular, in the case of "sugar" in "Feltwell-CHV," $K$-root allowed us to obtain a correlation coefficient higher $(+1.18 \%)$ than the one given by GGR, although, in the cases of "carrots" and "potatoes," GGR performed slightly better than $K$-root (Table IV).

We note that GGR-MoLC obtained slightly lower estimation accuracies when applied to the single "Feltwell" classes than when applied to the whole image areas in the "Feltwell" data set. This may be interpreted as a consequence of the fact that the number of samples used to compute a class-conditional PDF estimate is significantly smaller than the number of samples available to generate the PDF estimate for the whole image (namely $250 \cdot 350=87500)$.

In any case, GGR-MoLC allowed us to obtain good estimates when applied both to large data sets, representing whole image areas, and to smaller data sets, including only the samples drawn from given classes. This suggests a good stability of the GGR-MoLC procedure to the number of samples. In order to further investigate this issue, a specific experiment was carried out: Focusing, for instance, on the "stubble" class in "Feltwell-CHV," the set of 2441 samples available for this class was iteratively sub-sampled (removing, at each iteration, about $10 \%$ of the samples) and the GGR-MoLC procedure was applied to the subsampled data set. As shown in Fig. 9, the correlation coefficient remained above $96 \%$, even if the sample size was reduced to about $50 \%$ of the original size, above $94 \%$ after a $72 \%$ reduction, and above $90 \%$ even after an $80 \%$ reduction. Only when the sample size was reduced of more than $80 \%$ of the original size did the correlation coefficient decrease below $90 \%$, although it still remained above $85 \%$ even after an
$88 \%$ reduction. From an experimental viewpoint, this further suggests good stability properties of the GGR-MoLC estimation process (at least, on the considered data set) also in the finite sample-size situation (a consistency property regarding the asymptotic behavior of the estimator is described in Section III and Appendix B).

\section{CONCLUSION}

In this paper, an innovative parametric model has been proposed to characterize the statistics of the SAR complex backscattered signal, under the assumption of distributed backscattering. Specifically, a closed form has been derived for the resulting amplitude parametric PDF, and a parameter estimation algorithm, based on the Mellin transform and the method of log-cumulants, has been developed and tested on several real XSAR, E-SAR, ERS-1, and airborne SAR images.

The numerical experiments have proven that the proposed method outperforms the majority of the previously proposed approaches to SAR backscattering parametric modeling, namely, the Nakagami-Gamma model, the $K$ distribution, the "heavy-tailed" S $\alpha S$ generalized Rayleigh distribution, and two IG models. Specifically, the PDF estimates generated by the proposed GGR model almost always achieve higher correlation coefficients with the image histograms than the above-mentioned models, also exhibiting better visual fits between the estimates and the histograms themselves. Accurate results have been obtained, in particular, for images acquired by several different SAR sensors, thus proving the proposed parametric algorithm to be a flexible and effective estimation tool. We note that good estimation accuracies have also been obtained for multilook SAR data (although multilooking issues are not explicitly addressed in the methodological development of the proposed algorithm), thus confirming the flexibility and applicability of the algorithm to a wide variety of radar images.

Similar results have also been obtained by applying the proposed method to the modeling of the statistics of the gray levels drawn from single land-cover classes, thus suggesting the effectiveness of the model in the applications to both homogeneous sets of samples and to whole images, possibly representing strongly heterogeneous areas with many land-cover typologies. As a future development of the method, the integration of GGR into a finite mixture density scheme [64] could be considered in order to allow one to identify a proper GGR model for each land-cover class in an image, thus further improving the PDF estimation accuracy.

Visually biased estimates are obtained for very "peaky" empirical distributions, hence suggesting, as a possible development, a further generalization of the proposed model, for instance, by assuming different values for the parameters of the generalized Gaussian distributions characterizing the real and imaginary components of the complex signal. Such a choice would yield an even higher modeling flexibility, although at the expense of a much more complicated parameter estimation process. 
The method has turned out not to be suitable for all the considered images, since the estimation process can yield no solutions for specific combinations of the values of the samplelog-cumulants. However, in this respect, the experiments have suggested a complementarity between the proposed model and the $K$ distribution, since, for almost all the considered images, only one of these two models can be feasibly estimated. In addition, the resulting GGR or $K$ model always better fits the image histogram than the Nakagami-Gamma model, the $\mathrm{S} \alpha \mathrm{S}$ generalized Rayleigh model, or the two $\mathcal{G}^{h}$ and RiIG IG models, although these two recent models obtain, for some of the images, results very similar to the ones provided by GGR or $K$-root. In any case, the experiments have pointed out that GGR exhibits a wider applicability than the $K$ distribution, since GGR has turned out to be feasible for eight of the ten images used, whereas the $K$ distribution has been feasible for only three images. As a future development of this research activity, the observed complementarity may be further exploited by performing a pre-analysis of the image histogram in order to automatically check on the feasibilities of the GGR and $K$ models and, in the case of a joint applicability of both PDFs, in order to select the optimal model for the input SAR image.

Concerning the MoLC estimation process developed for GGR, good asymptotic properties are guaranteed theoretically, as the estimator is proven to be consistent. A good behavior has also been noted experimentally with regard to the finite-sample-size case, as the accuracy of the estimates generated by GGR-MoLC turned out to be significantly stable with respect to (even large) reductions of the data set employed by the estimation process.

All the considered MoLC-based parametric estimation strategies require very short computation times, as the nonlinear equations associated with the method of log-cumulants can be easily solved, for instance, by using a simple bisection method. In particular, the $K$ model involves the numerical solution of a system of two nonlinear equations, whereas the other three parametric families require, at most, the numerical solution of a single equation [29]. However, as discussed in [21], the numerical computations required by the $K$ model are not critical, thus, not taking much longer estimation times than the other three PDFs.

In any case, a further reduction in the computation time for GGR could be obtained by specifically optimizing the numerical computations of the PDF expression and of the $G_{\nu}(\cdot)$ function $(\nu=0,1,2)$. In this paper, standard numerical integration techniques [59] have been used to compute the integrals involved by these quantities. As in the case with the usual special functions (e.g., the Gamma function or the Bessel functions), we expect a reduction in the computation time if we adopt more sophisticated numerical strategies, like case-specific series expansions [59]. Finally, we stress that the computation time is almost independent of the image size, since the sample-log-cumulants can be computed by directly using the image histogram. Hence, the number of pixels in the image influences only the time taken to compute the histogram, but not the time required to estimate the values of the parameters that optimally fit the histogram itself.

\section{APPENDIX A \\ COMPUTATIONS OF THE MELlin TRANSFORM AND OF THE LOG-CUMULANTS OF THE GGR MODEL}

By plugging expression (14) in the definition (20) of the Mellin transform, and by introducing the auxiliary parameter $\lambda$ and the auxiliary function $A(\cdot, \cdot)$ (both defined in Section III-B), we obtain

$$
\begin{aligned}
\phi_{r}(s)= & \mathcal{M}\left(p_{r}\right)(s)=\frac{\gamma^{2} c^{2}}{\Gamma^{2}\left(\frac{1}{c}\right)} \int_{0}^{+\infty} r^{s} \\
& \cdot \int_{0}^{\pi / 2} \exp \left[-(\gamma r)^{c}\left(|\cos \theta|^{c}+|\sin \theta|^{c}\right)\right] d \theta d r \\
= & \frac{\gamma^{2}}{\lambda^{2} \Gamma^{2}(\lambda)} \int_{0}^{+\infty} r^{s} \int_{0}^{\pi / 2} \exp \left[-(\gamma r)^{1 / \lambda} A(\theta, \lambda)\right] d \theta d r \\
s \in & \mathbb{C} .
\end{aligned}
$$

According to the Fubini-Tonelli theorem [58], the function $(r, \theta) \longmapsto r^{s} \exp \left[-(\gamma r)^{1 / \lambda} A(\theta, \lambda)\right]$ is Lebesgue-integrable on $[0,+\infty) \times[0, \pi / 2]$, thus allowing an exchange of the order of integration, i.e.,

$\phi_{r}(s)=\frac{\gamma^{2}}{\lambda^{2} \Gamma^{2}(\lambda)} \int_{0}^{\pi / 2} d \theta \int_{0}^{+\infty} r^{s} \exp \left[-(\gamma r)^{1 / \lambda} A(\theta, \lambda)\right] d r$

The substitution $r \longmapsto \xi=(\gamma r)^{1 / \lambda} A(\theta, \lambda)$ yields

$$
\begin{aligned}
\phi_{r}(s)= & \frac{\gamma^{2}}{\lambda^{2} \Gamma^{2}(\lambda)} \\
& \cdot \int_{0}^{\pi / 2} d \theta \int_{0}^{+\infty} \frac{\xi^{\lambda s}}{\gamma^{s} A(\theta, \lambda)^{\lambda s}} \\
& \cdot \exp (-\xi) \frac{\lambda \xi^{\lambda-1}}{\gamma A(\theta, \lambda)^{\lambda}} d \xi \\
= & \frac{1}{\lambda \gamma^{s-1} \Gamma^{2}(\lambda)} \int_{0}^{+\infty} \xi^{\lambda s+\lambda-1} \\
& \cdot \exp (-\xi) d \xi \int_{0}^{\pi / 2} \frac{d \theta}{A(\theta, \lambda)^{\lambda s+\lambda}} .
\end{aligned}
$$

Expressing the first integral as a Gamma function [24] eventually yields the following expression for the second-kind characteristic function of the GGR model

$$
\phi_{r}(s)=\frac{\Gamma(\lambda s+\lambda)}{\lambda \gamma^{s-1} \Gamma^{2}(\lambda)} \int_{0}^{\pi / 2} \frac{d \theta}{A(\theta, \lambda)^{\lambda s+\lambda}} .
$$

According to (33), $\phi_{r}$ is defined in a neighborhood of 1 , thus allowing the definition of log-cumulants and the consequent application of MoLC. The second-kind second characteristic function turns out to be

$$
\begin{aligned}
\psi_{r}(s)= & \ln \phi_{r}(s) \\
= & \ln \Gamma(\lambda s+\lambda)-\ln \lambda-(s-1) \ln \gamma \\
& -2 \ln \Gamma(\lambda)+\ln \int_{0}^{\pi / 2} \frac{d \theta}{A(\theta, \lambda)^{\lambda s+\lambda}} .
\end{aligned}
$$

According to the definition of the digamma and polygamma functions [29], we obtain

$$
\begin{aligned}
& \psi_{r}^{\prime}(s)=\lambda \Psi(\lambda s+\lambda)-\ln \gamma \\
& -\lambda \int_{0}^{\pi / 2} \frac{\ln A(\theta, \lambda)}{A(\theta, \lambda)^{\lambda s+\lambda}} d \theta\left[\int_{0}^{\pi / 2} \frac{d \theta}{A(\theta, \lambda)^{\lambda s+\lambda}}\right]^{-1}
\end{aligned}
$$


and

$$
\begin{aligned}
\psi_{r}^{\prime \prime}(s)= & \lambda^{2} \Psi(1, \lambda s+\lambda) \\
& +\lambda^{2} \int_{0}^{\pi / 2} \frac{\ln ^{2} A(\theta, \lambda)}{A(\theta, \lambda)^{\lambda s+\lambda}} d \theta \\
& \cdot\left[\int_{0}^{\pi / 2} \frac{d \theta}{A(\theta, \lambda)^{\lambda s+\lambda}}\right]^{-1} \\
& -\lambda^{2}\left[\int_{0}^{\pi / 2} \frac{\ln A(\theta, \lambda)}{A(\theta, \lambda)^{\lambda s+\lambda}} d \theta\right]^{2} \\
& \cdot\left[\int_{0}^{\pi / 2} \frac{d \theta}{A(\theta, \lambda)^{\lambda s+\lambda}}\right]^{-2} .
\end{aligned}
$$

Thus, introducing the integral function (26), we obtain the following expressions for the first and second log-cumulants of the GGR distribution

$$
\begin{aligned}
& \kappa_{1}=\psi_{r}^{\prime}(1)=\lambda \Psi(2 \lambda)-\ln \gamma-\lambda \frac{G_{1}(\lambda)}{G_{0}(\lambda)} \\
& \kappa_{2}=\psi_{r}^{\prime \prime}(1)=\lambda^{2} \Psi(1,2 \lambda)+\lambda^{2} \frac{G_{2}(\lambda) G_{0}(\lambda)-G_{1}(\lambda)^{2}}{G_{0}(\lambda)^{2}} .
\end{aligned}
$$

\section{APPENDIX B}

\section{ProOF OF THE CONSISTENCY OF THE PROPOSED ESTIMATOR}

Here, we study the asymptotic properties of the developed estimator, and prove its consistency. Since the estimation procedure involves explicitly the parameter $\lambda=1 / c$ instead of $c$ itself, without loss of generality we refer to $(\lambda, \gamma)$, not to $(c, \gamma)$, as the parameter vector of the GGR distribution. Dealing with the asymptotic behavior, we assume the availability of a random sequence $\left\{r_{n}\right\}_{n=1}^{\infty}$ of i.i.d. data samples, drawn according to a GGR distribution. We denote by $\Theta:(0,+\infty)^{2} \rightarrow \mathbb{R}^{2}$ the nonlinear transformation that maps a parameter vector $\xi=(\lambda, \gamma)$ into the resulting $\log$-cumulant vector $\kappa=\left(\kappa_{1}, \kappa_{2}\right)$, i.e.,

$$
\Theta(\lambda, \gamma)=\left(\lambda \Psi(2 \lambda)-\ln \gamma-\lambda \frac{G_{1}(\lambda)}{G_{0}(\lambda)}, H(\lambda)\right) .
$$

Hence, denoting by $\xi^{*}=\left(\lambda^{*}, \gamma^{*}\right)$ the true parameter vector, the resulting true log-cumulants of the data distribution are the components of the vector $\kappa^{*}=\Theta\left(\xi^{*}\right)=\Theta\left(\lambda^{*}, \gamma^{*}\right)$. These log-cumulants can be estimated according to the data $\left\{r_{n}\right\}_{n=1}^{\infty}$ by introducing the sequence $\left\{\hat{\kappa}_{n}=\left(\hat{\kappa}_{1 n}, \hat{\kappa}_{2 n}\right)\right\}_{n=1}^{\infty}$ of samplemean and sample-variance estimates, i.e.,

$$
\hat{\kappa}_{1 n}=\frac{1}{n} \sum_{k=1}^{n} \ln r_{k}, \quad \hat{\kappa}_{2 n}=\frac{1}{n-1} \sum_{k=1}^{n}\left(\ln r_{k}-\hat{\kappa}_{1 n}\right)^{2} .
$$

Thanks to the strict monotonicity of $H(\cdot)$ and to the invertibility of (24) with respect to $\gamma, \Theta(\cdot)$ is an invertible mapping, that yields, for any $n=1,2, \ldots$, a unique solution $\hat{\xi}_{n}=\left(\hat{\lambda}_{n}, \hat{\gamma}_{n}\right)=\Theta^{-1}\left(\hat{\kappa}_{n}\right)$ of the vector equation $\Theta\left(\xi_{n}\right)=\hat{\kappa}_{n}$. In addition, both $\Theta$ and its inverse $\Theta^{-1}$ turn out to be continuous mappings, in particular, $\Theta^{-1}$ being continuous at $\kappa^{*}$. Therefore, for any $\varepsilon>0$, there exists $\delta_{\varepsilon}>0$ such that ${ }^{4}\left\|\kappa-\kappa^{*}\right\|_{2}<\delta_{\varepsilon}$ yields $\left\|\Theta^{-1}(\kappa)-\xi^{*}\right\|_{\infty}<\varepsilon$. Hence, if $\left\|\hat{\kappa}_{n}-\kappa^{*}\right\|_{2}<\delta_{\varepsilon}$, then $\left\|\hat{\xi}_{n}-\xi^{*}\right\|_{\infty}<\varepsilon$, which yields the following relation among events:

$$
\left\{\left\|\hat{\kappa}_{n}-\kappa^{*}\right\|_{2}<\delta_{\varepsilon}\right\} \subset\left\{\left\|\hat{\xi}_{n}-\xi^{*}\right\|_{\infty}<\varepsilon\right\} .
$$

Therefore

$$
\begin{aligned}
P\left\{\left|\hat{\lambda}_{n}-\lambda^{*}\right|<\varepsilon,\left|\hat{\gamma}_{n}-\gamma^{*}\right|<\varepsilon\right\} & =P\left\{\left\|\hat{\xi}_{n}-\xi^{*}\right\|_{\infty}<\varepsilon\right\} \\
& \geq P\left\{\left\|\hat{\kappa}_{n}-\kappa^{*}\right\|_{2}<\delta_{\varepsilon}\right\} .
\end{aligned}
$$

Applying first the Markov inequality to the nonnegative random variable $\left\|\hat{\kappa}_{n}-\kappa^{*}\right\|_{2}$ [20] and then the Cauchy-Schwartz inequality [20], we have

$$
\begin{aligned}
P\left\{\left\|\hat{\kappa}_{n}-\kappa^{*}\right\|_{2}<\delta_{\varepsilon}\right\} & \geq 1-\frac{E\left\{\left\|\hat{\kappa}_{n}-\kappa^{*}\right\|_{2}\right\}}{\delta_{\varepsilon}} \\
& \geq 1-\frac{E\left\{\left\|\hat{\kappa}_{n}-\kappa^{*}\right\|_{2}^{2}\right\}^{1 / 2}}{\delta_{\varepsilon}} .
\end{aligned}
$$

Therefore

$$
\begin{aligned}
& P\left\{\left|\hat{\lambda}_{n}-\lambda^{*}\right|<\varepsilon,\left|\hat{\gamma}_{n}-\gamma^{*}\right|<\varepsilon\right\} \\
& \quad \geq 1-\frac{E\left\{\left\|\hat{\kappa}_{n}-\kappa^{*}\right\|_{2}^{2}\right\}^{1 / 2}}{\delta_{\varepsilon}} \\
& \quad=1-\frac{E\left\{\left(\hat{\kappa}_{1 n}-\kappa_{1}^{*}\right)^{2}+\left(\hat{\kappa}_{2 n}-\kappa_{2}^{*}\right)^{2}\right\}^{1 / 2}}{\delta_{\varepsilon}} .
\end{aligned}
$$

The sample-mean estimate $\hat{\kappa}_{1 n}$ of $\kappa_{1}^{*}=E\{\ln r\}$ and the sample-variance estimate $\hat{\kappa}_{2 n}$ of $\kappa_{2}^{*}=\operatorname{Var}\{\ln r\}$ are known to be unbiased, with variances given by [20]

$$
\operatorname{Var}\left\{\hat{\kappa}_{1 n}\right\}=\frac{\kappa_{2}^{*}}{n}, \quad \operatorname{Var}\left\{\hat{\kappa}_{2 n}\right\}=\frac{1}{n}\left(\beta-\frac{n-3}{n-1}\left(\kappa_{2}^{*}\right)^{2}\right)
$$

where $\beta=E\left\{\left(\ln r-\kappa_{1}^{*}\right)^{4}\right\}$. Therefore

$$
\begin{aligned}
& P\left\{\left|\hat{\lambda}_{n}-\lambda^{*}\right|<\varepsilon,\left|\hat{\gamma}_{n}-\gamma^{*}\right|<\varepsilon\right\} \\
& \quad \geq 1-\frac{\left[\operatorname{Var}\left\{\hat{\kappa}_{1 n}\right\}+\operatorname{Var}\left\{\hat{\kappa}_{2 n}\right\}\right]^{1 / 2}}{\delta_{\varepsilon}} \\
& \quad=1-\frac{1}{\delta_{\varepsilon} \sqrt{n}}\left(\kappa_{2}^{*}+\beta-\frac{n-3}{n-1}\left(\kappa_{2}^{*}\right)^{2}\right)^{1 / 2} .
\end{aligned}
$$

Finally, we conclude that

$$
\lim _{n \rightarrow+\infty} P\left\{\left|\hat{\lambda}_{n}-\lambda^{*}\right|<\varepsilon,\left|\hat{\gamma}_{n}-\gamma^{*}\right|<\varepsilon\right\}=1
$$

which proves that the random vector sequence $\left\{\left(\hat{\lambda}_{n}, \hat{\gamma}_{n}\right)\right\}_{n=1}^{\infty}$ converges in probability to the true parameter vector $\left(\lambda^{*}, \gamma^{*}\right)$. Hence, the developed MoLC estimator for the proposed GGR distribution turns out to be consistent [57].

\section{ACKNOWLEDGMENT}

The authors would like to thank the French Space Agency CNES and the French Research Center CESBIO, which provided the "Bourges," "Flevoland," "Suisse-Lake," and "Oberp-

${ }^{4}$ We denote here by $\|\cdot\|_{2}$ and $\|\cdot\|_{\infty}$ the Euclidean and the Tchebitchev (i.e., uniform) norm on $\mathbb{R}^{2}$, respectively [65]. Adopting the former in the domain of the mapping $\Theta^{-1}$ and the latter in the co-domain is advantageous in the calculations and involves no loss of generality, as all norms on a finite-dimensional vector space are equivalent [65]. 
faffenhofen" data sets, all available on the CD-ROM "Speckle filters compararative tests" (copyright CNES, 2001). The authors would also like to thank the anonymous reviewers for their constructive criticism.

\section{REFERENCES}

[1] M. Cheney, "A mathematical tutorial on synthetic aperture radar," SIAM Rev., vol. 43, no. 2, 2001.

[2] — , "An introduction to synthetic aperture radar (SAR) and SAR interferometry," in Approximation Theory X: Wavelets, Splines, and Applications, C. K. Chui, L. L. Schumacher, and J. Stockler, Eds. Nashville, TN: Vanderbilt Univ. Press, 2002, pp. 167-177.

[3] C. Oliver and S. Quegan, Understanding Synthetic Aperture Radar Images. Norwood, MA: Artech House, 1998.

[4] V. S. Frost and L. S. Yurovsky, "Maximum likelihood classification of synthetic aperture radar imagery," Comput. Vis., Graph., Image Process., vol. 32, pp. 291-313, 1985.

[5] Y. Kouskoulas, F. T. Ulaby, and L. E. Pierce, "The Bayesian hierarchical classifier (BHC) and its application to short vegetation using multifrequency polarimetric SAR," IEEE Trans. Geosci. Remote Sens., vol. 42, no. 2, pp. 469-477, Feb. 2004.

[6] A. N. Nyonngui, E. Tonye, and A. Akono, "Evaluation of speckle filtering and texture analysis methods for land cover classification from SAR images," Int. J. Remote Sens., vol. 23, no. 9, pp. 1895-1925, 2002.

[7] R. O. Duda, P. E. Hart, and D. G. Stork, Pattern Classification, 2nd ed. New York: Wiley, 2001

[8] R. Touzi, "A review of speckle filtering in the context of estimation theory," IEEE Trans. Geosci. Remote Sens., vol. 40, no. 11, pp. 2392-2404, Nov. 2002.

[9] J. S. Lee, "Speckle analysis and smoothing of synthetic aperture radar images," Comput. Graph. Image Process., vol. 17, pp. 24-32, 1981.

[10] V. S. Frost, J. A. Stiles, K. S. Shanmugan, and J. C. Holtzman, "A model for radar images and its application to adaptive digital filtering for multiplicative noise," IEEE Trans. Pattern Anal. Mach. Intell., vol. PAMI-4, no. 2, pp. 157-167, Feb. 1982

[11] C. Tison, J.-M. Nicolas, F. Tupin, and H. Maitre, "A new statistical model for Markovian classification of urban areas in high-resolution SAR images," IEEE Trans. Geosci. Remote Sens., vol. 42, no. 10, pp. 2046-2057, Oct. 2004

[12] E. Parzen, "On estimation of probability density function and mode," Signal Process., vol. 33, pp. 267-281, 1962.

[13] C. M. Bishop, Neural Networks for Pattern Recognition, 2nd ed. Oxford, U.K.: Oxford Univ. Press, 1996.

[14] L. Bruzzone, M. Marconcini, U. Wegmuller, and A. Wiesmann, "An advanced system for the automatic classification of multitemporal SAR images," IEEE Trans. Geosci. Remote Sens., vol. 42, no. 6, pp. 1321-1334, Jun. 2004

[15] P. Mantero, G. Moser, and S. B. Serpico, "Partially supervised classification of remote sensing images using SVM-based probability density estimation," in Proc. IEEE Workshop Advances in Techniques for Analysis of Remotely Sensed Data, Oct. 2003, pp. 327-336.

[16] V. N. Vapnik, Statistical Learning Theory. New York: Wiley, 1998.

[17] J. Weston, A. Gammerman, M. Stitson, V. Vapnik, V. Vovk, and C. Watkins, "Support vector density estimation," in Advances in Kernel Methods Support Vector Learning, B. Schölkopf, C. J. C. Burges, and A. J. Smola, Eds. Cambridge, MA: MIT Press, 1999, pp. 293-306.

[18] J. W. Goodman, "Some fundamental properties of speckle," J. Opt. Soc. Amer, vol. 66, pp. 1145-1150, 1977.

[19] E. E. Kuruoglu and J. Zerubia, "Modeling SAR images with a generalization of the Rayleigh distribution," IEEE Trans. Image Process., vol. 13, no. 4, pp. 527-533, Apr. 2004.

[20] A. Papoulis, Probability, Random Variables, and Stochastic Processes, 3rd ed. New York: McGraw-Hill, 1991.

[21] J. M. Nicolas. (2002) Introduction Aux Statistiques De Deuxième Espéce: Application Aux Lois D'images RSO [Online]. Available: http://www.tsi.enst.fr/

[22] R. Dana and D. Knepp, "The impact of strong scintillation on space based radar design II: Noncoherent detection," IEEE Trans. Aerosp. Electron. Syst., vol. AES-22, no. 1, pp. 34-46, Jan. 1986.

[23] J. W. Goodman, "Statistical properties of laser speckle patterns," in Laser Speckle and Related Phenomena. Heidelberg, Germany: Springer-Verlag, 1975, pp. 9-75.
[24] W. Rudin, Principles of Mathematical Analysis, 2nd ed. New York: McGraw-Hill, 1976.

[25] I.-R. Joughin, D. P. Winebrenner, and D. B. Percival, "Probability density functions for multilook polarimetric signatures," IEEE Trans. Geosci. Remote Sens., vol. 32, no. 3, pp. 562-574, May 1994.

[26] G. K. Karagiannidis, D. A. Zogas, and S. A. Kotsopoulos, "On the multivariate Nakagami-m distribution with exponential correlation," IEEE Trans. Commun., vol. 51, no. 8, pp. 1240-1244, Aug. 2003.

[27] J.-S. Lee, K. W. Hoppel, S. A. Mango, and A. R. Miller, "Intensity and phase statistics of multilook polarimetric and interferometric SAR imagery," IEEE Trans. Geosci. Remote Sens., vol. 32, no. 5, pp. 1017-1028, Sep. 1994.

[28] J.-M. Nicolas and F. Tupin, "Gamma mixture modeled with second kind statistics: Application to SAR image processing," presented at the IGARSS Conf., Toronto, ON, Canada, 2002.

[29] J.-M. Nicolas, "Introduction aux statistiques de deuxième espéce: Applications des log-moments et des log-cumulants a l'analyze des lois d'images radar" (in French), Traitement du Signal, vol. 19, 2002.

[30] C. Tison, J.-M. Nicolas, and F. Tupin, "Accuracy of fisher distributions and log-moment estimation to describe histograms of high-resolution SAR images over urban areas," presented at the IGARSS Conf., Toulouse, France, Jul. 21-25, 2003.

[31] P. Levy, Calcul des Probabilites (in French). Paris, France: GauthiersVillars, 1925.

[32] E. E. Kuruoglu, "Density parameter estimation of skewed $\alpha$-stable distributions," IEEE Trans. Signal Process., vol. 49, no. 10, pp. 2192-2201, Oct. 2001

[33] E. E. Kuruoglu and J. Zerubia, "Skewed $\alpha$-stable distributions for modeling textures," Pattern Recognit. Lett., vol. 24, pp. 339-348, 2003.

[34] F. Bowman, Introduction to Bessel Functions. New York: Dover, 1968.

[35] I. Sneddon, The Use of Integral Transforms. New York: McGraw-Hill, 1972.

[36] E. Jakeman and P. N. Pusey, "A model for non-Rayleigh sea echo," IEEE Trans. Antennas Propagat., vol. AP-24, no. 6, pp. 806-814, Nov. 1976.

[37] — "Significance of $K$ distributions in scattering experiments," Phys Rev. Lett., vol. 40, pp. 546-550, 1978.

[38] C. J. Oliver, "A model for non-Rayleigh scattering statistics," Opt. Acta, vol. 31, no. 6, pp. 701-722, 1984.

[39] J. Jao, "Amplitude distribution of composite terrain radar clutter and the $K$-distribution," IEEE Trans. Antennas Propagat., vol. AP-32, no. 10, pp. 1049-1052, Oct. 1984.

[40] Y. Delignon and W. Pieczynski, "Modeling non-Rayleigh speckle distribution in SAR images," IEEE Trans. Geosci. Remote Sens., vol. 40, no. 6, pp. 1430-1435, Jun. 2002.

[41] C. J. Oliver, "Correlated $K$-distributed scattering model," Opt. Acta, vol. 32, pp. 1515-1547, 1985.

[42] S. H. Yueh and J. A. Kong, " $K$ distribution and polarimetric terrain radar clutter," J. Electromagn. Waves Applicat., vol. 3, no. 8, pp. 747-768, 1999.

[43] A. C. Frery, H.-J. Muller, C. C. F. Yanasse, and S. Sant'Anna, "A model for extremely heterogeneous clutter," IEEE Trans. Geosci. Remote Sens., vol. 35, no. 3, pp. 648-659, May 1997.

[44] H.-J. Muller and R. Pac, "G-statistics for scaled SAR data," in Proc. IEEE Geoscience and Remote Sensing Symp., vol. 2, 1999, pp. 1297-1299.

[45] T. Eltoft, "Speckle modeling and filtering," presented at the NORSIG Symp., 2003.

[46] M. Sekine and Y. Mao, Weibull Radar Clutter. London, U.K.: IEE Press, 1990.

[47] W. Szajnowski, "Estimators of log-normal distribution parameters," IEEE Trans. Aerosp Electron. Syst., vol. AES-13, no. 5, pp. 533-536, Sep. 1977.

[48] Y. Delignon, A. Marzouki, and W. Pieczynski, "Estimation of generalized mixtures and its application to image segmentation," IEEE Trans. Image Process., vol. 6, no. 10, pp. 1364-1375, Oct. 2001.

[49] J.-M. Nicolas and A. Maruani, "Lower-order statistics: A new approach for probability density functions defined on $\mathbb{R}^{+}$, , presented at the EUSIPCO Conf., Tampere, Finland, 2000.

[50] F. Bujor, E. Trouve, L. Valet, J.-M. Nicolas, and J.-P. Rudant, "Application of log-cumulants to the detection of spatiotemporal discontinuities in multitemporal SAR images," IEEE Trans. Geosci. Remote Sens., vol. 42, no. 10, pp. 2073-2084, Oct. 2004.

[51] A. Tesei and C. S. Regazzoni, "HOS-based generalized noise PDF models for signal detection optimization," Signal Process., vol. 65, pp. 267-281, 1998. 
[52] C. Bouman and K. Sauer, "A generalized gaussian image model for edge-preserving map estimation," IEEE Trans. Image Process., vol. 2, no. 3, pp. 296-310, Jul. 1993.

[53] P. Moulin and J. Liu, "Analysis of multiresolution image denoising schemes using generalized Gaussian and complexity priors," IEEE Trans. Inf. Theory, vol. 45, no. 3, pp. 909-919, Apr. 1999.

[54] S. Mallat, A Wavelet Tour of Signal Processing. New York: Academic, 1999.

[55] E. P. Simoncelli, "Statistical models for images: Compression restoration and synthesis," in Proc. Asilomar Conf. Signals, Systems and Computers, vol. 1, Lausanne, Switzerland, 1997, pp. 673-678.

[56] E. P. Simoncelli and E. H. Adelson, "Noise removal via bayesian wavelet coring," in Proc. 3rd IEEE Int. Conf. Image Processing, vol. 1, 1996, pp. 379-382.

[57] H. L. Van Trees, Detection, Estimation and Modulation Theory. New York: Wiley, 1968, vol. 1.

[58] W. Rudin, Real and Complex Analysis, 3rd ed. New York: McGrawHill, 1987.

[59] W. H. Press, S. A. Teukolsky, W. T. Wetterling, and B. P. Flannery. (2002) Numerical Recipes in C. Cambridge Univ. Press, Cambridge, U.K. [Online]. Available: http://www.library.cornell.edu/nr/bookcpdf.html

[60] S. B. Serpico, L. Bruzzone, and F. Roli, "An experimental comparison of neural and statistical nonparametric algorithms for supervised classification of remote sensing images," Pattern Recognit. Lett., vol. 17, pp. 1331-1341, 1996.

[61] G. Moser, J. Zerubia, and S. B. Serpico. (2004) SAR Amplitude Probability Density Function Estimation Based on a Generalized Gaussian Scattering Model. INRIA, Sophia Antipolis, France. [Online]. Available: http://www.inria.fr/rrt/rr-5153.html

[62] M. Datcu, K. Seidel, and M. Walessa, "Spatial information retrieval from remote sensing images: Part I. Information theoretical perspective," IEEE Trans. Geosci. Remote Sens., vol. 36, no. 5, pp. 1431-1445, Sep. 1998.

[63] M. Petrou, F. Giorgini, and P. Smits, "Modeling the histograms of various classes in SAR images," Pattern Recognit. Lett., vol. 23, pp. 1103-1107, 2002.

[64] R. A. Redner and H. F. Walker, "Mixture densities, maximum likelihood, and the EM algorithm," SIAM Rev., vol. 26, no. 2, pp. 195-239, 1984.

[65] W. Rudin, Functional Analysis, 2nd ed. New York: McGraw-Hill, 1990.

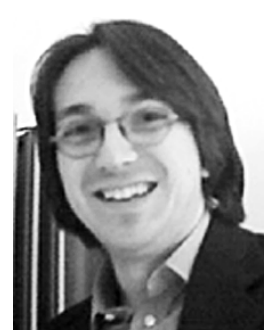

Gabriele Moser (S'03-M'05) received the Laurea (M.S.) degree (summa cum laude) in telecommunications engineering and the Ph.D. degree in space sciences and engineering from the University of Genoa, Genoa, Italy, in 2001 and 2005, respectively.

Since 2001, he has cooperated with the Signal Processing and Telecommunications Research Group (SP\&T), Department of Biophysical and Electronic Engineering (DIBE), University of Genoa, in the field of remote-sensing image analysis. From January to March 2004, he was a visiting student with the Institut National de Recherche en Informatique et en Automatique (INRIA), Sophia Antipolis, France, working with the "Ariana" research group on the problem of SAR data modeling. He is currently a Research Fellow at DIBE. His research activity is focused on image-processing and image-analysis methodologies for remote-sensing data interpretation. In particular, his current research interests include SAR data analysis, multitemporal image classification, partially supervised classification, hyperspectral image analysis, and contextual classification. He has been a Reviewer for several international journals.

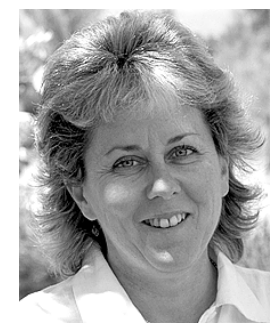

Josiane Zerubia (S'78-M'81-SM'99-F'03) received the M.Sc. degree from the Department of Electrical Engineering at ENSIEG, Grenoble, France, in 1981, and the Doctor of Engineering, $\mathrm{Ph} . \mathrm{D}$., and the Habilitation degrees from the University of Nice, Sophia-Antipolis, France, in 1986, 1988, and 1994, respectively.

She has been a Permanent Research Scientist at the Institut National de Recherche en Informatique et en Automatique (INRIA), Sophia Antipolis, France, since 1989, and Director of Research since July 1995.

She was Head of the PASTIS Remote Sensing Laboratory (INRIA Sophia-Antipolis) from mid-1995 to 1997. Since January 1998, she has been head of the Ariana research group (INRIA/CNRS/University of Nice), which also works on remote sensing. She has been Adjunct Professor at Sup'Aero (ENSAE), Toulouse, France, since 1999. Before that, she was with the Signal and Image Processing Institute, University of Southern California (USC), Los Angeles, as a Postdoctorate. She was also a Researcher with the LASSY (University of Nice/CNRS), from 1984 to 1988, and with the Research Laboratory of Hewlett Packard in France and in Palo Alto, CA, from 1982 to 1984. Her current research interests are in image processing using probabilistic models and variational methods. She also works on parameter estimation and optimization techniques.

Dr. Zerubia was a member of the IEEE IMDSP Technical Committee (SP Society) from 1997 to 2003; Associate Editor of the IEEE TRANSACTIONS ON IMAGE PROCESSING from 1998 to 2002; Guest Co-Editor of a special issue of the IEEE TRANSACTIONS ON PATTERN ANALYSIS AND MACHINE INTELLIGENCE in 2003; and member-at-large of the Board of Governors of the IEEE SP Society from 2002 to 2004. She has been an Area Editor of IEEE TRANSACTIONS ON IMAGE PROCESSING since 2003. She has also been a member of the editorial board of the French Society for Photogrammetry and Remote Sensing (SFPT) since 1998 and of the International Journal of Computer Vision since 2004. She has been a member of the IEEE BISP Technical Committee (SP Society) since 2005. She was Co-Chair of two workshops on Energy Minimization Methods in Computer Vision and Pattern Recognition (EMMCVPR'01, Sophia Antipolis, and EMMCVPR'03, Lisbon, Portugal); Co-Chair of a workshop on Image Processing and Related Mathematical Fields (IPRM'02, Moscow, Russia); and Chair of a workshop on Photogrammetry and Remote Sensing for Urban Areas, Marne La Vallee, France, 2003.

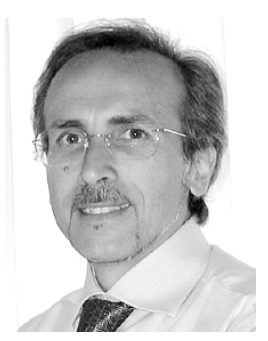

Sebastiano B. Serpico (S'87-SM'00) received the Laurea degree in electronic engineering and the Ph.D. degree in telecommunications from the University of Genoa, Genoa, Italy, in 1982 and 1989, respectively.

Since 1982, he has cooperated with the Department of Biophysical and Electronic Engineering (DIBE), University of Genoa, in the field of image processing and recognition. He was an Assistant Professor from 1990 to 1998 and an Associate Professor of telecommunications from 1998 to 2004 with the Faculty of Engineering, University of Genoa, where he taught signal theory, pattern recognition, telecommunication systems, and electrical communications. He is currently a Full Professor of telecommunications. From 1995 to 1998, he was Head of the Signal Processing and Telecommunications Research Group (SP\&T), Department of Biophysical and Electronic Engineering (DIBE), University of Genoa, and is currently Head of the SP\&T Laboratory. He is the Chairman of the Institute of Advanced Studies in Information and Communication Technologies. His current research interests include the application of pattern recognition (feature selection, classification, change detection, and data fusion) to remotely sensed images. He is the author or coauthor of more than 150 scientific publications, including journals and conference proceedings.

Dr. Serpico was the recipient of the Recognition of TGARS Best Reviewers from the IEEE Geoscience and Remote Sensing Society in 1998. He co-edited a special issue of the IEEE TRANSACTIONS ON GEOSCIENCE AND REMOTE SENSING on the subject of the analysis of hyperspectral image data (July 2001) and a special issue on the advances in techniques for the analysis of remotely sensed data (March 2005). Since 2001, he has been an Associate Editor of the TRAnsactions on Geoscience ANd REMOte SENSING. He is a member of the International Association for Pattern Recognition Society (IAPR). 